\title{
Macrophages stimulate epicardial VEGFaa expression to trigger cardiomyocyte proliferation in larval zebrafish heart regeneration
}

Finnius A. Bruton ${ }^{1 *}$, Aryan Kaveh ${ }^{1}$, Katherine M. Ross-Stewart ${ }^{1}$, Gianfranco Matrone ${ }^{1}$, Magdalena E.M. Oremek², Emmanouil G. Solomonidis ${ }^{1}$, Carl S. Tucker ${ }^{1}$, John J. Mullins $^{1}$, Mairi Brittan ${ }^{1}$, Jonathan M. Taylor ${ }^{3}$, Adriano G. Rossi ${ }^{2}$, Martin A. Denvir ${ }^{1}$.

${ }^{1}$ Centre for Cardiovascular Science, Queen's Medical Research Institute, University of Edinburgh, Edinburgh, United Kingdom.

${ }^{2}$ Centre for Inflammation Research, Queen's Medical Research Institute, University of Edinburgh, Edinburgh, United Kingdom.

${ }^{3}$ Department of Physics, University of Glasgow, Glasgow, United Kingdom.

Corresponding author: *

\section{Abstract}

Cardiac injury induces a sustained macrophage response in both zebrafish and mammals. Macrophages perform a range of both beneficial and detrimental functions during mammalian cardiac repair, yet their precise roles in zebrafish cardiac regeneration are not fully understood. Here we characterise cardiac regeneration in the rapidly regenerating larval zebrafish laser injury model and use macrophage ablation and macrophage-null irf8 mutants to define the role of macrophages in key stages of regeneration. Live heartbeat-synchronised imaging and RNA sequencing revealed an early proinflammatory phase, marked by tnfa+ macrophages, which then resolved to an anti-inflammatory, profibrotic phase. Macrophages were required for cardiomyocyte proliferation but not for functional or structural recovery following injury. Importantly, we found that macrophages are specifically recruited to the epicardialmyocardial niche, triggering the expansion of the epicardium which upregulates VEGFaa expression to induce cardiomyocyte proliferation. Hence, revealing a novel mechanism by which macrophages facilitate cardiac regeneration.

\section{Introduction}


33 Zebrafish are highly regenerative, exhibiting the capacity to restore full structure and function to a wide range of tissues following injury ${ }^{1-5}$. Cardiac injury is one such example where adult mammals are only able to facilitate maladaptive repair but zebrafish exhibit full tissue regeneration ${ }^{6,7}$. In humans, the most severe form of cardiac injury is myocardial infarction (MI), where occlusion of a coronary artery triggers ischemic injury to the myocardium, leading to the loss of approximately 1 billion cardiomyocytes $^{8}$. Adult mammalian cardiomyocytes are considered largely postmitotic, switching to hypertrophic growth shortly after birth. They are therefore unable to restore lost myocardium, which is instead replaced with non-contractile scar tissue ${ }^{9}$. Consequently, MI patients suffer sequalae of maladaptive remodelling, leading to left ventricular dilation and thinning of the scar, further decreasing the function of the heart ${ }^{10,11}$. Hence, there is a need for medical innovation which can reverse or prevent this process.

In contrast to mammalian models of $\mathrm{MI}$, apical resection and cryoinjury MI models in zebrafish show full regeneration of lost myocardium via the dedifferentiation and proliferation of surviving cardiomyocytes ${ }^{12,13}$. Cardiac regeneration is complex and dynamic, with zebrafish hearts undergoing debridement of dead myocardium, followed by transient fibrosis, revascularisation and eventual replacement of cardiomyocytes ${ }^{14}$. The inflammatory response has been demonstrated to be crucial for each of these key events, both in zebrafish and also in other regenerative species such as axolotls and neonatal mice ${ }^{15-17}$. In particular, macrophages have emerged as important cellular regulators of tissue regeneration. Indeed, macrophage ablation has been shown to abrogate regeneration across multiple organs and organisms, including the adult zebrafish heart ${ }^{15,16,18}$. However, the precise contribution of macrophages to cardiac repair has been complicated by disparate results following macrophage perturbation in mouse models of $\mathrm{MI}$, where macrophages have been reported to be both beneficial and detrimental ${ }^{7,19,20}$. This is in part attributed to substantial heterogeneity of macrophage subtypes, and phenotypic plasticity ${ }^{21,22}$. Recent studies have confirmed the presence of macrophage subsets in zebrafish, yet their functional niche and interactions with other key cell types of the heart, such as the epicardium, remain poorly understood ${ }^{23,24}$. The larval zebrafish model of cardiac regeneration offers a 
66

67

68

69

70

rapidly than adults, occurring in just 48 hours after cardiac laser injury in 3-day old larvae ${ }^{25,26}$. Combined with their amenability for live in vivo imaging and genetic tractability, this model becomes a powerful tool with which to carefully examine how macrophages support multiple aspects of cardiac regeneration.

Here we report an in-depth characterisation of the macrophage response and several key regenerative processes in larval zebrafish cardiac regeneration, finding the heart regeneration program between the larvae and adults to be highly conserved. Abolition of the macrophage response using metronidazole-nitroreductase ablation of macrophages or the macrophage null irf8 ${ }^{-/-}$mutant ${ }^{27}$, demonstrated a requirement for these cells in removal of apoptotic cells, epicardial activation and cardiomyocyte proliferation. Interestingly, we found that one of the ways macrophages exert their proproliferative effect is via epicardial VEGFaa and downstream endocardial notch signalling. Our study reveals that macrophages invade the epicardial-myocardial niche, inducing expansion of epicardial cell numbers which increases epicardial VEGFaa expression, leading to an upregulation of endocardial notch signalling and the cardiac developmental growth pathway.

\section{Results}

\section{Macrophages display cellular heterogeneity following cardiac injury}

We first assessed macrophage heterogeneity and recruitment dynamics following larval cardiac injury. We crossed the zebrafish pan-macrophage reporter line $T g$ (mpeg1:GFP) with $T g$ (csf1ra:gal4;UAS:mCherry-NfsB) (shortened here to csf1ra: mCherry) (Supplementary Figure 1a). Csf1ra (colony stimulated factor 1 receptor) is a cytokine required for macrophage development and used as a macrophage reporter promoter in mammals ${ }^{28}$.

Larval hearts were lasered at the ventricular apex at 72 hours post-fertilisation (hpf) and imaged at 2, 6, 24, and 48 hours post injury (hpi) (Figure 1a). Macrophages migrate to the injured ventricular apex within 2 hours, peak at 6 and maintain elevated numbers until $48 \mathrm{hpi}$ (Figures $1 \mathrm{~b} \& 1 \mathrm{c}$ ). We found that not all recruited macrophages were co-positive for both transgenes, leading to three subsets 1) mpeg1+csf1ra- 
100 (19.3 $\pm 5.1 \%), 2)$ mpeg1-csf1ra+ (2.8 $\pm 2.1 \%)$ and 3) mpeg1+csf1ra+ $(77.9 \pm 5.7 \%)$.

101 Similar dynamics were seen for subsets 1 \& 3 but since mpeg1-csf1ra+ were

102 exceedingly rare it is not possible to know if the dynamics are likewise similar. Both

103 subsets exhibit a range of morphologies with no overt difference between groups

104 (Figure 1d, Video 1). Importantly, our data demonstrate that larval macrophages

105 recruited to cardiac injury are heterogenous in their marker expression, similar to adult

106 zebrafish $^{29}$, and suggest a comparatively complex macrophage response in the larval

107 model.

\section{Macrophages display cellular plasticity following cardiac injury}

111 To examine if macrophages display plasticity and convert to an inflammatory

112 phenotype in the larval cardiac injury model, we performed cardiac laser injury on

113 Tg(tnfa:GFP;mpeg1:mCherry) larvae. (Figure 1e \& 1f). Quantification of tnfa+

114 macrophage number revealed a transient tnfa+ subset $(19.3 \pm 4.9 \%$ of mpeg $1+$

115 macrophages, $n=24$ ), found only at the 24 hpi timepoint and rarely in uninjured larvae

116 (Figure 1f \& Supplementary figure 1b). We also observed that from $24 \mathrm{hpi}$,

117 macrophages retract their pseudopods and become spherical, further suggesting a

118 shift in phenotype (Supplementary figure 1e).

120 We reasoned that if tnfa+ macrophages were indeed inflammatory macrophages then

121 application of M1-polarisation cytokine IFN- $\gamma$ would increase their abundance. A single

122 intravenous injection of zebrafish recombinant protein IFN- $\gamma$-rel, immediately prior to

123 cardiac injury, increased the proportion of $\operatorname{tnf} \alpha+m p e g 1+$ macrophages from

$12426.4 \pm 11.0 \%$ in PBS injected controls to $78.8 \pm 9.5 \%$, supporting the suggestion that

125 these were inflammatory macrophages (Figure 1g \& Supplementary figure 1c \& 1d).

127 Furthermore, in vivo imaging live in the beating heart showed recruited macrophages

128 becoming tnfa:GFP+ after arrival at the injured ventricle, confirming that this represents true in situ conversion (Figure 1h, Video 2). Taken together, these data

130 show that, as in adults, macrophages display plasticity and become inflammatory in 131 response to cardiac injury, confirming the complexity of the macrophage response in 132 this model. 
134 Larval cardiac laser lesions are similar in structure to adult cryoinjury

136 To validate analyses of macrophage function in the larval injury model, we first sought

137 to determine if the laser lesion is comparable to adult cryoinjury and mammalian 138 infarcts. Using the line $\operatorname{Tg}(m y / 7: m K a t e C A A X ; m y / 7: h 2 b-G F P)$, which labels 139 cardiomyocyte sarcolemma and chromatin respectively, we observed that, following 140 injury, a circlet of cardiomyocytes with pyknotic nuclei formed (Figure 2a). These 141 pyknotic nuclei were TUNEL + at $6 \mathrm{hpi}$, confirming apoptosis and they encircled the 142 GFP- lesion (Figure 2b \& 2c). Heartbeat-synchronised LSFM (lightsheet fluorescence 143 microscopy) ${ }^{30}$ showed that nuclear condensation occurred extremely rapidly, being 144 identified by $1.5 \mathrm{hpi}$ (Supplementary figure 2a, Video 3).

146 We hypothesised that the GFP- epicentre of the laser lesion may contain cells that immediately necrose upon injury. To test this, we labelled necrotic cells by injecting propidium iodide $(\mathrm{PI})$ intravenously immediately following injury ( $<0.5 \mathrm{hpi})$. We found that there were indeed $\mathrm{PI}+$ cells in the GFP- region, and $\mathrm{PI}+$ debris scattered across the proximal myocardium from 1 hpi (Figure 2d). Time-lapse imaging of Pl-injected, injured Tg(mpeg1:GFP;myl7:h2b-GFP;myl7:mKateCAAX) hearts showed that necrotic cells are rapidly cleared within the first 0-2 hpi (Supplementary figure 2b \& Video 3). Necrotic cells either disintegrated or were squeezed out from the myocardium into the pericardial cavity, independently of macrophage contact (Video 4). Overall, this characterisation confirms the structure of the laser lesion mirrors the necrotic infarct and apoptotic border zone observed in adult zebrafish cryoinjury and mammalian $\mathrm{MI}^{14,31}$.

Macrophages contribute to the removal of apoptotic cardiomyocytes following injury

We next sought to understand what role macrophages play in the regeneration of the larval heart, which occurs within only 48 hours of the initial injury ${ }^{25,26}$. We used two different methods to induce macrophage-less hearts. Firstly we used the Tg(csf1ra:gal4;UAS:mCherry-NfsB) line (abbreviated hereafter to csf1ra:NfsBmCherry) that expresses a nitroreductase enzyme NfsB in macrophages, which 
167 induces cell-specific apoptosis when exposed to prodrug metronidazole ${ }^{32}$

168 (Supplementary figure 3a-d). Macrophage ablation only occurs in larvae expressing 169 the nitroreductase (NTR) and in the presence of metronidazole (NTR+met+).

170 Therefore, larvae only expressing the nitroreductase (NTR+met-) or only in the 171 presence of metronidazole (NTR-met+) are used as macrophage-replete control 172 groups. The second method was the use of the macrophage-null irf8-/- mutant ${ }^{27}$, IRF8 173 being a transcription factor required for macrophage development (Supplementary 174 figure $3 e-h)$.

176 To determine if macrophages are required for the removal of apoptotic cells, we 177 performed TUNEL staining on $\mathrm{irf}^{-/-}$and $i r f 8^{+/+} \operatorname{Tg}(m y / 7: h 2 b-G F P)$ larvae at the 178 standard 2, 6, 24, 48 hpi timepoints (Figure $2 \mathrm{e} \& 2 \mathrm{~g}$ ). In injured $\mathrm{irf}^{+/+}$hearts, the 179 number of apoptotic cardiomyocytes significantly increased at 2 hpi and 6 hpi compared to uninjured controls $(4.1 \pm 0.9$ vs $0.0 \pm 0.0$ and $5.3 \pm 1.0$ vs $0.1 \pm 0.1$ respectively, $n=15-29$ ) but returned to baseline by $24 \mathrm{hpi}$. However, although injured macrophage-null irf8 ${ }^{-/-}$hearts showed a similar initial pattern of cell death at 2 hpi and

1836 hpi $(5.5 \pm 0.8$ \& $5.9 \pm 0.9$ apoptotic cardiomyocytes respectively), apoptotic 184 cardiomyocyte cells were still present at $24 \mathrm{hpi}$, only returning to uninjured levels by $48 \mathrm{hpi}$.

In the macrophage ablation model, we saw a similar pattern of results where the numbers of apoptotic cells were negligible in uninjured hearts of all treatment groups, but peaked at $6 \mathrm{hpi}$ following injury (NTR+met-, NTR-met+ \& NTR+met+ $=6.4 \pm 0.5$, $6.4 \pm 0.5$ and $6.0 \pm 0.5, n=10-12$ ) (Figure $2 f \& 2 h$ ). By $24 \mathrm{hpi}$ the non-ablated groups no longer possessed significantly increased numbers of TUNEL+ myocardial cells; however, the macrophage-ablated group showed a retention of apoptotic cells at 24 hpi $(\mathrm{NTR}+$ met $+=1.5 \pm 0.3)$ that resolved by $48 \mathrm{hpi}$.

To verify that macrophages are directly removing myocardial debris, we performed time-lapse imaging of injured Tg(myl7:GFP;mpeg1:mCherry) larvae. We observed small GFP+ pieces of myocardial debris near the GFP- lesion being removed and internalised by macrophages (Figure 2i \& Supplementary Figure 2c \& Video 5), confirming the essential role of macrophages in lesion debridement. 


\section{Macrophages are not obligatory for structural or functional recovery of the larval} heart

Next, we sought to investigate if macrophages are required for structural and functional recovery of the larval heart following laser-injury. We injured $T g(m y / 7: G F P)$ larvae following macrophage ablation and acquired serial 3D scans of the cardiac structure of individual larvae by heartbeat-synchronised LSFM (Figure 3a). In all treatment groups, the lesion size was consistent between $2 \mathrm{hpi}$ and $6 \mathrm{hpi}$, with no difference between groups. By $24 \mathrm{hpi}$ the lesion had almost completely regressed (95\% to $\left.37.1 \mu m^{2} \pm 24.4, n=11-22\right)$ in macrophage-replete NTR+met- larvae (Figure 3b). However, for larvae in the macrophage-ablated NTR+met+ and the other macrophage-replete NTR-met+ group, lesion closure was slightly delayed at $24 \mathrm{hpi}$

213 (73\% and $75 \%$ to $234.7 \pm 59.7 \mu \mathrm{m}^{2}$ and $221.4 \pm 84.6 \mu \mathrm{m}^{2}$ respectively). By $48 \mathrm{hpi}$ the

214 lesions of larvae from each group had entirely regressed and luminal surface renders

215 of injured ventricles showed normal trabecular structure (Figure 3a). These results suggest macrophages are not required for lesion closure, but that metronidazoletreatment slightly delays this process.

Using $T g$ (myl7:GFP) larvae, we acquired lateral-view videos of beating hearts with epifluorescence microscopy and tested if macrophage ablation affected recovery of cardiac function. Immediately following injury at $2 \mathrm{hpi}$, volumetric ejection fraction was decreased in all groups from $74 \%$ in uninjured ventricles to $54 \%$ in injured (Figure 3c \& Supplementary figure $4 \mathrm{a} \& 4 \mathrm{~b})$. Ejection fraction recovered quickly by $6 \mathrm{hpi}$ in all treatment groups, ( $78 \%$ injured vs $\sim 87 \%$ uninjured) and by $24 \mathrm{hpi}$ and 48 hpi injured hearts were functionally indistinguishable from uninjured hearts. These data suggest that injured larval hearts recover their function rapidly, and that this recovery is not macrophage dependent.

We next performed identical experiments examining the recovery of cardiac structure and function with $\mathrm{Tg}(\mathrm{my} / 7: \mathrm{GFP})$ larvae on an irf8 mutant background. Both $\mathrm{irf} 8^{+/+}$and

231 irf8-/- genotype larvae showed substantial lesion regression ( $80 \%)$ between $6 \mathrm{hpi}$ and 
$\mu m^{2} \pm 59.5$, respectively) (Figure $3 d \& 3 e$ ). No difference in lesion size was seen at any

234 timepoint and both genotypes had completely closed their lesions by 48 hpi. Normal trabecular structure was seen in both groups at $48 \mathrm{hpi}$ following full structural recovery (Figure $3 d$ ). The recovery of ejection fraction in this model followed the same trend as that of the metronidazole-nitroreductase model, with the ejection fraction of injured larvae being indistinguishable from uninjured larvae by $24 \mathrm{hpi}$ in both genotypes (Figure 3f). Our near identical findings in the irf8 macrophage null model confirm that larval hearts rapidly recover following laser injury and that this process is macrophage independent.

Finally, we wished to understand the mechanism of lesion closure. We performed heartbeat-synchronised time-lapse imaging of lesions in $T g(m y / 7: G F P)$ larvae immediately following injury. We observed GFP+ myocardial budding on opposite sides of the lesion border zone and subsequent invasion into the lesion, adhering to each other to form bridges (Figure $3 \mathrm{~g}$, Video 6). Repeating this experiment in Tg(myl7:h2b-GFP;myl7:mKateCAAX) larvae facilitated the tracking of individual cardiomyocytes by virtue of their labelled nuclei and plasma membranes (Video 7 \& Supplementary figure $2 \mathrm{~d}$ ). We found that cardiomyocytes bordering the lesion did not divide but extended protrusions into the lesion until they adhered with other single cardiomyocytes bridging from the opposing side of the lesion. These imaging insights suggest that myocardial structure is first restored by morphogenesis rather than cell division.

Macrophage ablation abolishes an injury-associated increase in cardiomyocyte proliferation

To test if cardiomyocyte proliferation increases in response to laser-injury, we performed EdU staining in $T g(m y l 7: h 2 b-G F P)$ larvae in two experiments. In the first experiment, uninjured and injured larvae were exposed to EdU during 0-24 hpi and then at 24-48 hpi for the second (Figure 4a). Comparison between uninjured and injured hearts revealed no significant difference in the proportion of EdU+ cardiomyocyte nuclei $0-24$ hpi $(21.3 \pm 3.3$ vs $18.9 \pm 3.4$ respectively, $n=10-14)$ (Figure 
proportion of EdU+ cardiomyocytes in injured hearts relative to uninjured $(43.5 \pm 1.8 \%$ vs $32.2 \pm 2.0 \%$ respectively, $n=17-25)$. Time-lapse in vivo imaging of dividing cardiomyocytes showed nuclear division followed by cytokinesis, exclusively gives rise to mononuclear cells, with no obvious hypertrophy (Video 8, Supplementary Figure $5 a)$.

To understand if macrophages are required for the injury-dependent increase in cardiomyocyte proliferation, EdU was pulsed during the proliferative 24-48 hpi window in the macrophage-less models (Figure 4d). In the metronidazole-nitroreductase ablation model we found that the percentage of EdU+ cardiomyocytes increased in injured hearts in both the NTR+met- and NTR-met+ control groups, but not in the macrophage-ablated NTR+met+ group (Figure $4 \mathrm{e} \& 4 \mathrm{f}$ ). This result indicates that macrophages are a requirement for injury-dependent increase in cardiomyocyte proliferation. However, in contrast to the metronidazole-nitroreductase ablation model, analysis of cardiomyocyte proliferation in irf8 ${ }^{--}$mutants revealed that they too significantly increased the percentage of EdU+ cardiomyocytes following injury, comparably to $i r f 8^{+/+}$larvae (Figure $4 \mathrm{~g} \& 4 \mathrm{~h}$ ).

To resolve this disparity, we examined more closely the differences between these models. We found, like others ${ }^{18}$, that irf8 ${ }^{-/}$mutants possess a greater global number of neutrophils than observed in $i r f 8^{+/+}$fish and mount a larger neutrophil response to injury (Supplementary Figure $5 b \& 5 c$ ). Since we do not observe an increased neutrophil response in NTR+met+ larvae, we hypothesised that neutrophils might be compensating for macrophages in irf8 ${ }^{-/-}$larvae (Supplementary Figure $5 \mathrm{~d}$ ). To test this hypothesis, we inhibited neutrophil recruitment in irf8-- larvae using the receptor antagonist 'SB225002' which blocks CXCR1/2 activation, a key chemokine receptor for neutrophil migration. CXCR1/2 inhibition successfully lowered the number of recruited neutrophils $(2.0 \pm 3.4$ vs $0.43 \pm 0.18)$ and abolished the injury-associated increase in cardiomyocytes in irf8 ${ }^{-/}$(Supplementary Figure $5 \mathrm{e}-\mathrm{g}$ ). Taken together, this suggests that macrophages are required for cardiomyocyte proliferation but can be substituted by excess neutrophils. 
Next, we sought to understand which biological processes might still be occurring by the final 48 hpi timepoint of the larval cardiac injury model. We performed RNAseq on pooled, uninjured and injured larval hearts at $48 \mathrm{hpi} \mathrm{(Figure} \mathrm{5a).} \mathrm{We} \mathrm{found} 418$ genes were upregulated ( $\log _{2}$ fold change $>1$ ), and 1,046 downregulated in injured hearts. We did not observe differential expression of markers of proliferation such as MCM2, mKi67 and PCNA, suggesting that the proliferation we observe from $24 \mathrm{hpi}$ is concluded by $48 \mathrm{hpi}$ (Figure $5 \mathrm{~b}$ ). In agreement with this, gene ontology analysis indicated categories such as growth factors and cell proliferation not to be enriched at 48 hpi (Supplementary Figure 6e).

Most inflammatory and M1 markers were either not differentially expressed or were downregulated in injured hearts, such as $\| 1 \mathrm{~b}$ (Figure $5 \mathrm{~b} \&$ Supplementary file 1). In contrast, we found injury-associated upregulation of 39 collagen isoforms, several profibrotic genes such as $\operatorname{tgfb} 1 \mathrm{a}$ and markers of epithelial to mesenchymal transition (EMT) such as vimentin. Similarly, hierarchical clustering of differentially expressed genes revealed 9 distinct clusters with Cluster 1 being upregulated in injured hearts and enriched in collagens, matrix metalloproteins (MMPs) and fibroblast growth factors (FGFs) (Figure 5c, Supplementary file 2). Additionally, Cluster 2 contained several EMT genes, Cluster 8 genes relating to cell recruitment and lymphangiogeneis whilst

Cluster 7 contained several embryonic-associated myosins and myosin binding proteins such as myl10 and cald1b. Clusters 3-6 \& 9 were downregulated in injury, Cluster 2 was enriched for immune genes and Clusters 4 and 6 for growth factors, with Clusters 3 and 5 having no clear identity. Taken together, our RNAseq results suggest that the inflammatory and proliferative stages are largely concluded by $48 \mathrm{hpi}$ and that a pro-resolving and reparative phase dominates thereafter.

\section{Cardiac injury induces epicardial activation and VEGFaa upregulation}

Our detailed characterisation of the larval laser injury model revealed a macrophagedependent, cardiomyocyte proliferative response occurring at 24-48 hpi. We therefore utilised the rapidity and imaging opportunities offered by the model to investigate the 
underlying mechanism of the induction of cardiomyocyte proliferation. Epicardial

333 VEGFaa has recently been demonstrated to drive cell cardiomyocyte proliferation in

334 adult zebrafish following cryoinjury and we hypothesised the same mechanism might drive cardiomyocyte proliferation in the injured larval heart ${ }^{33}$.

We found robust vegfaa:GFP expression specifically in mesothelial cells overlying the myocardium (Figure 6a). Colocalisation with established epicardial marker tcf21 in uninjured Tg(tcf21:DsRed;vegfaa:GFP) larvae confirmed these cells to be early epicardium (Figure 6b). Next we investigated if epicardial vegfaa:GFP expression changes following injury by 3D fluorescence intensity analysis of uninjured and regenerating hearts. We found that epicardial vegfaa:GFP intensity increased significantly at $48 \mathrm{hpi}$ (Figure 6c \& 6d). Interestingly, this was due both to an increase in the number of epicardial cells and their individual intensity suggesting the epicardium activates and responds to injury by both proliferation and gene expression changes to increase VEGFaa (Supplementary figure 7c \& 7d).

Macrophages localise to the epicardial niche and induce the expansion of epicardial cell number

Given that our data showed that macrophage ablation abolishes injury-dependent cardiomyocyte proliferation (Figure 5f), we hypothesised that macrophages might be required for epicardial activation. To test this hypothesis, we ablated macrophages and assessed if epicardial activation still occurred at $48 \mathrm{hpi}$. Following injury we observed increased vegfaa:GFP expression in both macrophage-replete NTR-met+ and NTR+met- groups, but not in macrophage ablated NTR+met+ hearts (Figure $6 f$ \& $6 \mathrm{~g})$. Interestingly, macrophage ablation did not affect vegfaa:GFP expression per cell, but did block the expansion of epicardial cell number following injury (Figure 6h \& Supplementary Figure 7e). Furthermore, 3D analysis of macrophage localisation following injury showed that recruited macrophages invade the myocardial-epicardial niche and synapse with epicardial cells (Figure 6e). Importantly, macrophage or neutrophil vegfaa:GFP expression was not observed at any timepoint (Supplementary Figure $7 a \& 7 b)$. Our data therefore strongly suggest that the recruitment of macrophages to the epicardium is essential for subsequent epicardial activation, thus increasing net cardiac vegfaa expression. 
VEGFaa is both required and sufficient for cardiomyocyte proliferation in larval zebrafish

To verify if epicardial VEGFaa was driving cardiomyocyte proliferation in larval cardiac regeneration, we first tested if VEGFaa was sufficient to stimulate cardiomyocyte proliferation. Recombinant zebrafish VEGFaa protein (zfVEGFaa) was intravenously microinjected into the circulation of $72 \mathrm{hpf} T g$ (myl7:h2b-GFP) larvae and total cardiomyocyte number assessed at 24 and $48 \mathrm{hpt}$ (hours post-treatment) (Figure 7a). zfVEGFaa increased total cardiomyocyte number by $13.3 \%$ relative to PBS-injected controls at 24 hpt (Figure 7b \& 7c).

To test if VEGF signalling is required for injury-associated cardiomyocyte proliferation, we used a high-affinity, pan-VEGFR receptor antagonist AV951 (Tivozanib) to block VEGF signalling ${ }^{34}$. We bathed larvae in 10nM AV951 over the course of our cardiac injury model, pulsed with EdU at 24-48 hpi and quantified EdU+ cardiomyocytes at 48 hpi (Figure 7d). Interestingly, AV951 decreased the proportion of EdU+ cardiomyocytes in both the uninjured and injured groups (uninjured $38.4 \pm 3.4$ vs $28.0 \pm 3.0$ and injured $42.9 \pm 3.8$ vs $31.6 \pm 2.2, n=13-36$ ) (Figure $7 e \& 7 f)$. Together, these data suggest that VEGF signalling in the heart is driving cardiomyocyte proliferation in the larval heart, both as part of normal development and following cardiac injury.

\section{Notch and Nrg-ErbB signalling are required for cardiomyocyte proliferation}

We next investigated if macrophage-induced epicardial VEGFaa signalling could be interacting with more established effectors of cardiomyocyte proliferation. Notch and $\mathrm{Nrg}-\mathrm{ErbB}$ were strong candidates as both are required for adult heart regeneration and cardiomyocyte proliferation in adult zebrafish ${ }^{35-38}$. We first verified if these signalling pathways were required for cardiomyocyte proliferation in the larval heart following injury. We laser-injured $T g(m y l 7: n / s D s R e d)$ larvae, and bathed them in $100 \mu \mathrm{M}$ of pan-notch inhibitor DAPT ((N-[N-(3,5-difluorophenacetyl)-I-alanyl]-Sphenylglycine t-butyl ester) (Figure $7 \mathrm{~g}$ ). DAPT is a gamma secretase inhibitor and has been demonstrated in both zebrafish and drosophila to phenocopy notch mutants ${ }^{39-}$ 
41. Notch signalling inhibition decreased cardiomyocyte number by $\sim 8 \%$ in uninjured hearts $(253.3 \pm 5.2$ vs $233.4 \pm 4.5, \mathrm{n}=25-38)$ and $\sim 14 \%$ injured hearts $(266.0 \pm 5.6$ vs 229.5 $\pm 3.6, n=25-38)$. (Figure $7 \mathrm{~h} \&$ $7 \mathrm{i})$.

We repeated this experiment, with $1.75 \mu \mathrm{M}$ ErbB2 antagonist AG1478 (Figure 7g). Small molecule inhibitor AG1478 selectively inhibits ErbB2, a required co-receptor for ErbB4 dimerization and subsequent neuregulin signal transduction and faithfully phenocopies erbb2 mutants ${ }^{42}$. Interestingly, the results exactly replicated those of the notch signalling inhibition experiment, decreasing cardiomyocyte number by $\sim 9 \%$ in uninjured $(257.2 \pm 5.8$ vs $235.2 \pm 4.2)$ and by $\sim 13 \%$ in injured hearts $(265.7 \pm 5.5$ vs $229.5 \pm 5.9$ ) (Figure 7j \& 7k). These results confirm that both notch signalling and NrgErbB signalling are required for the expansion of cardiomyocyte number in both uninjured and injured larval hearts.

\section{Cardiac injury and VEGFaa induce endocardial notch signalling}

Given the individual requirement of VEGF, notch and Nrg-ErbB signalling for cardiomyocyte proliferation in the larval heart, we sought to understand if these signalling components might act in one pathway. Previous studies have demonstrated developmental larval zebrafish heart growth to be activated by cardiac contraction, via endocardial-notch>Nrg-ErbB signalling ${ }^{43,44}$. We hypothesised that VEGFaa might be driving cardiomyocyte proliferation by increasing endocardial notch signalling and consequently augmenting this developmental pathway (Figure 8a).

To test if VEGFaa could activate endocardial notch signalling, we utilised the notch signalling reporter line $T g(T p 1: v e n u s-P E S T)$ as a readout of cardiac notch signalling. Recombinant zfVEGFaa injected into $3 \mathrm{dpf}$ larvae, and their hearts were analysed via heart-synchronised light-sheet microscopy at 6, 24 and 48 hpt (Figure 8b). Furthermore, an additional group of larvae were injected with zfVEGFaa but also bathed in ErbB2 antagonist AG1478. According to our hypothesised pathway (Figure 8a), we reasoned that zfVEGFaa injection should upregulate notch signalling but that inhibition of Nrg-ErbB signalling should be unable to suppress zfVEGFaa-induced notch upregulation. 
433 Notch signalling was primarily in the endocardium, colocalising with endothelial 434 reporter kdrl:mCherry but was relatively low intensity and only detectable in a subset 435 of larvae at any given timepoint (Figure 8c). We found, zfVEGFaa injection increased 436 the percentage of larvae with notch+ (Tp1:Venus+) endocardium $(46.4 \%$ to $78.6 \%$, $437 \mathrm{n}=28$ ) at $6 \mathrm{hpi}$ but not at the 24 and $48 \mathrm{hpi}$ timepoints (Figure 8d). Furthermore, 438 AG1478 failed to block the increase in the percentage of notch+ hearts following zfVEGFaa injection, confirming Nrg-ErbB signalling was not upstream of vegfaa or notch signalling. In fact, zfVEGFaa+AG1478 treated larvae had a substantially higher percentage of notch + hearts at $48 \mathrm{hpi}$ than those treated with zfVEGFaa alone $(25.0 \%$ vs $0 \%$ ). This is suggestive of a negative feedback mechanism, supporting previous findings of Nrg-ErbB being downstream of notch signalling ${ }^{43}$.

To test if this pathway (Figure 8a) acted similarly in injury, we substituted zfVEGFaa injection for cardiac injury and repeated the experiment (Figure 8e). Cardiac injury similarly increased the percentage of hearts possessing notch+ endocardium but this occurred later, at $48 \mathrm{hpi}(50.0 \%$ vs $11.1 \%, \mathrm{n}=18)$ (Figure $8 \mathrm{f} \& 8 \mathrm{~g})$. As before, AG1478 did not block notch signalling, rather it seemed to enhance it. Whilst the percentage of notch+ hearts in the injured group did not significantly increase by $24 \mathrm{hpi}$, injured+AG1478 treated larvae did significantly increase relative to uninjured larvae $(52.9 \%$ vs $11.1 \%)$.

Taken together, these results demonstrate that cardiac injury and VEGFaa increase endocardial notch signalling, providing a novel mechanism whereby macrophages can trigger cardiomyocyte proliferation via stimulation of epicardial vegfaa expression.

\section{Discussion}

In this study we have presented the first detailed characterisation of the larval zebrafish model of heart regeneration, demonstrating the heterogeneity and plasticity of macrophages in cardiac injury and testing the requirement of macrophages for the removal of apoptotic cells, cardiomyocyte proliferation, epicardial activation and recovery of cardiac structure and function. Furthermore, we demonstrated the utility of the larval cardiac injury model by taking advantage of its in vivo cardiac imaging 
opportunities and amenability to pharmacological intervention to discover a novel role for macrophages in driving cardiomyocyte proliferation via epicardial activation.

Our examination of macrophages in larval zebrafish cardiac injury suggests that they may faithfully recapitulate the phenotypic complexity and function found in the adult cryoinjury model. As previously shown in adult hearts ${ }^{29}$, we detected mpeg1+csf1ra+ and mpeg1+csf1ra- macrophage subsets. We found these cells to have identical recruitment dynamics and no obvious differences in morphology or behaviour. Recent Cre-Lox lineage tracing has shown that mpeg1+csf1ra- cells have a nonhaematopoietic origin, are csfr1a-independent developmentally and, unlike mpeg1+csf1ra+ cells, are not phagocytic ${ }^{45,46}$. Future work should focus on understanding the precise roles of these subsets in cardiac regeneration, in particular mpeg1+csf1ra- macrophages.

In addition to macrophage heterogeneity, we observed macrophage phenotypic plasticity. We used heartbeat-synchronised live imaging to show that macrophages can convert from mpeg1+tnfa- to mpeg1+tnfa+. This is the first time that macrophage phenotype conversion has been imaged in the heart. Studies examining zebrafish macrophages in spinal cord and tail transection have demonstrated thfa to mark M1like macrophages, which then transition to M2-like macrophages ${ }^{24}$. Our success in increasing the percentage of tnfa+ macrophages by canonical M1-polarising cytokine IFN- $\gamma$-rel suggests that early tnfa+ macrophages are indeed proinflammatory. In agreement with findings in the in the adult cryoinjured heart ${ }^{29}$, we found this tnfa+ population of macrophages to be transient, only observed in the early response at 24 hpi. Similarly, our RNAseq data showed that, by $48 \mathrm{hpi,} \mathrm{injured} \mathrm{larval} \mathrm{hearts}$ downregulate inflammatory cytokines and growth factors but upregulate collagens and reparative cytokines. Our finding that a pro-resolving, fibrotic program is activated in injured hearts, despite full structural and functional recovery, is in agreement with a recent study showing the scar-deficient runx $1^{-/-}$zebrafish to undergo successful cardiac regeneration ${ }^{47}$. It might be that the fibrotic program is concomitantly activated upon resolution of inflammation, irrespective of a requirement for scar tissue.

We used two separate methods to examine the role of macrophages in larval heart regeneration. Interestingly, ablation via csf1ra-driven nitroreductase expression was 
still able to abolish numbers of csf1ra- macrophages at the injured heart. Possibly this is indicative of a positive-feedback system where csf1ra- macrophage recruitment is dependent on csf1ra+ macrophages. Cell death data acquired by either technique demonstrated that macrophages are required for the timely removal of apoptotic cells following injury. Interestingly, these apoptotic cells do eventually seem to be cleared even in the absence of macrophages. Our live imaging showed that dead cardiomyocytes can be expelled from the myocardium independently of macrophages. It is possible that this is a mechanical consequence of cardiac contraction, although a similar phenomenon is known to occur in neuroepithelium where neurons appear to extrude apoptotic cells out of tissue ${ }^{48}$.

Surprisingly, we found that the absence of macrophages did not affect the structural or functional recovery of the injured larval heart, despite macrophages being required for cardiomyocyte proliferation. This is in contrast to past studies where liposomal clodronate macrophage ablation and CCR2-antagonist inhibition of macrophage recruitment in regenerative neonatal mice, adult zebrafish and axolotl hearts causes blocked or delayed resolution of the infarct area ${ }^{15,16,49}$. The contrasting results in the larval heart might simply be a consequence of its small size and low transmural pressure, allowing surviving myocardium to rapidly 'knit' back together. Supporting this, we observed individual cardiomyocytes extending protrusions into the lesion.

520 Previous histological analysis of the border zone in injured adult zebrafish and neonatal mouse hearts has shown cardiomyocytes exhibiting a similar mesenchymal phenotype following partial dedifferentiation and disassembly of sarcomeres ${ }^{50,51}$. However, this is the first time this behaviour has been verified by time-lapse imaging live in a beating heart.

Studies in adult zebrafish and neonatal mice have shown ablation of macrophages to decrease cardiomyocyte proliferation ${ }^{15,17}$. However, early revascularisation is critical for cardiomyocyte proliferation and is macrophage-dependent, calling into question whether macrophages directly induce cardiomyocyte proliferation ${ }^{17,52}$. Larval hearts do not have supporting vasculature ${ }^{26}$; therefore our finding that cardiomyocyte proliferation is macrophage-dependent suggests macrophages facilitate cardiomyocyte proliferation by means other than revascularisation. Indeed, our data

533 indicate a novel mechanism whereby macrophages are recruited to the epicardial- 
myocardial niche and induce expansion of epicardial cell numbers and increase in the expression of mitogenic VEGFaa. This might explain previous findings in developing and injured mouse hearts where yolk-derived and Gata6+ pericardial cavity macrophages are recruited to the epicardium, respectively ${ }^{53,54}$ Future studies should seek to identify precisely how macrophages activate epicardium. Given we found that neutrophils can compensate for macrophages for cardiomyocyte proliferation, it is possible that a shared inflammatory factor triggers epicardial activation.

Endocardial notch signalling is required for cardiomyocyte proliferation in cryoinjured adults and myocardial growth by downstream Nrg1 in larvae ${ }^{43,55,56}$. Therefore, our finding that injury and VEGFaa increase endocardial notch signalling reveals an important mechanism whereby the epicardium can induce cardiomyocyte proliferation. In agreement with previous studies, we showed both notch and Nrg-ErbB signalling to be required for expansion of cardiomyocyte numbers in both uninjured and injured hearts ${ }^{57-59}$. Since we found VEGFaa inhibition decreased cardiomyocyte proliferation in uninjured larval hearts, it is likely that VEGFaa>notch>Nrg-ErbB is a developmental cardiac growth pathway that is upregulated upon injury and thus might be conserved in mammals. Our discovery that macrophages act upstream of this pathway therefore opens up exciting immunomodulatory opportunities for therapeutic enhancement of cardiac repair in the future.

\section{Materials and Methods}

\section{Zebrafish husbandry and lines used}

Zebrafish husbandry and maintenance was conducted as per standard operating procedures, in accordance with the Animals (Scientific Procedures) Act, 1986 and approved by The University of Edinburgh Animal Welfare and Ethical Review Board in a United Kingdom Home Office-approved establishment. All our experiments were performed on staged zebrafish aged between $3 \mathrm{dpf}$ and $5 \mathrm{dpf}$. The following transgenic and mutant lines were used: $\operatorname{Tg}(m y l 7: e G F P)^{t w u 26}$ 60, $\operatorname{Tg}(m p x: m C h e r r y)^{u w m 7}{ }^{61}$, Tg(mpeg1:mCherry)g/23 62, Tg(mpeg1:eGFP)gl22 62, (Tg(mpx:GFP) ${ }^{111463}, \operatorname{Tg}($ myl7:h2bGFP)zf52 64, Tg(myl7:mKateCAAX) ${ }^{S D 11}{ }^{65}, \mathrm{Tg}(\mathrm{fms}: G a 14 . V P 16)^{i 186}$, referred to as csfr1a:gal4 ${ }^{66}, T g(U A S-E 1 b: N f s B-m C h e r r y)^{c 264}$ abbreviated to UAS:NfsB-mCherry ${ }^{67}$, 
$\operatorname{Tg}\left(\right.$ vegfaa:eGFP) ${ }^{P D 260} \quad 33, \quad T g(m y l 7: n / s D s R e d)^{f 2} \quad 68 \quad T g(T N F a: e G F P)^{s a 43296 \quad 24}$, Tg(Tp1:venus-PEST) ${ }^{S 94069}$, Tg(kdrl:hsa.HRAS-mCherry) ${ }^{S 89670, T g(k d r l: G F P) / a 116 ~} 71$,

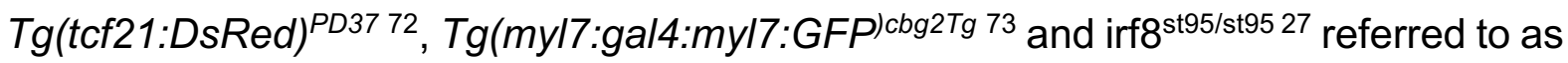
irf8 $^{-1}$. Tg(csf1ra:gal4:UAS:NfsB-mCherry) is abbreviated to csf1ra:NfsB-mCherry

572 throughout the manuscript for simplicity. Adults were day-crossed as appropriate to

573 yield desired combinations of transgenes in embryos. Embryos were treated with

$574 \quad 0.003 \%$ phenylthiourea (Fisher Scientific) at $7 \mathrm{hpf}$ to prevent pigment formation and therefore enhance image clarity. Embryos and larvae were incubated at $28.5^{\circ} \mathrm{C}$ in conditioned media/water $\left(6.4 \mathrm{mM} \mathrm{KCl}, 0.22 \mathrm{mM} \mathrm{NaCl}, 0.33 \mathrm{mM} \mathrm{CaCl}_{2} \cdot 2 \mathrm{H}_{2} \mathrm{O}, 0.33 \mathrm{mM}\right.$ $\left.\mathrm{MgSO} 4 \cdot 7 \mathrm{H}_{2} \mathrm{O}\right)+0.1 \%$ methylene blue $(\mathrm{w} / \mathrm{v})$ and imaged at room temperature $\left(23^{\circ} \mathrm{C}\right)$ using epifluorescence or light sheet fluorescence microscopy (details below). When necessary, larvae were anesthetized using $40 \mu \mathrm{g} / \mathrm{ml}$ tricaine methanesulfonate (Sigma Aldrich) in conditioned media.

\section{Cardiac laser injury}

A Zeiss Photo Activated Laser Microdissection (PALM) laser system (Zeiss) was used to precisely cause a localised injury at the ventricular apex of anesthetized $72 \mathrm{hpf}$ larvae $^{26}$. Larvae were mounted on a glass slide in $20 \mu$ lanesthetized conditioned media and lasered via a 20X objective. Injuries were deemed successful and complete once ventricular contractility decreased, the apex had shrunk, and the myocardial wall had swollen without causing cardiac rupture and subsequent bleeding. A successful cardiac injury results in the portion of dysfunctional tissue losing fluorescent myocardial transgenic fluorescence signal. Uninjured larvae were treated in the same manner up to the point of laser injury, when they were individually transferred into single wells of a 24-well plate and maintained in the same environmental conditions as injured fish.

\section{Epifluorescence microscopy}

Larvae were mounted laterally in conditioned media on a glass slide and imaged using a Leica M205 FA stereomicroscope with GFP and mCherry filters. For all serial timepoint epifluorescence imaging experiments, number of immune cells on the heart 
601 were quantified by manually observing and counting cells moving synchronously with the beating heart. Heart images were acquired using $2 X 0.35 \mathrm{NA}$ objective.

603

\section{Heart-synchronised light-sheet microscopy}

Individual larvae were prepared for light sheet fluorescence microscopy (LSFM) by embedding in 1\% low melting-point agarose (ThermoFisher) in anesthetized conditioned media inside FEP tubes (Adtech Polymer Engineering). Agar embedding prevents gradual drift of the embryo in the FEP tube, without causing developmental perturbations during long-term imaging. Larvae were used only once for a time-lapse imaging experiment, and any repeats shown come from distinct individuals. Larvae were mounted head down such that the heart faces toward both illumination and imaging objectives to improve image clarity. All LSFM experiments were performed at room temperature $\left(23^{\circ} \mathrm{C}\right)$. Camera exposure times ranged from $5-15 \mathrm{~ms}$, laser excitation power was $11 \mathrm{~mW}$ and scans were performed at 3-5 minute intervals. Brightfield images acquired at $80 \mathrm{fps}$ were analysed in real-time to enable opticallygated acquisition of fluorescence $z$ slices at a set phase of cardiac contraction, usually mid diastole. The setup of our custom-built LSFM system has been previously reported

619 in detail ${ }^{30}$.

\section{Metronidazole-nitroreductase macrophage ablation model}

In order to selectively ablate macrophages prior to cardiac injury, embryos were

624 incubated as previously described until $48 \mathrm{hpf}$ and then treated as follows. Embryos were carefully dechorionated at $48 \mathrm{hpf}$ and screened based on fluorescence and split into groups appropriate to the experiment, for example larvae were always split into csf1ra:gal4;UAS:NfsB-mCherry+ and csf1ra:gal4;UAS:NfsB-mCherry-. Embryos were then transferred to either conditioned water or a $0.5 \mathrm{mM}$ metronidazole (Thermo Fisher Scientific) solution, both solutions also contained $0.003 \%$ phenylthiourea (Thermo Fisher Scientific) and 0.2\% DMSO (Sigma Aldrich). Larvae were then incubated in these solutions in the dark at $28.5^{\circ} \mathrm{C}$ for 24 hours prior to injury at $72 \mathrm{hpf}$. Larvae were then removed from the metronidazole solution and vehicle solution and placed in fresh conditioned water $+0.003 \%$ phenylthiourea for the remainder of the experiment. As 
shown in Figure 2, this is sufficient to ablate macrophages prior to injury and completely block subsequent macrophage recruitment to the injured heart.

\section{Neutral red staining}

Larvae were incubated at $72 \mathrm{hpf}$ in $5 \mu \mathrm{g} / \mathrm{mL}$ neutral red in conditioned water for 5 hours in the dark at $28.5^{\circ} \mathrm{C}$. Larvae were then washed twice for 5 minutes in conditioned water, anaesthetised with $40 \mu \mathrm{g} / \mathrm{ml}$ tricaine methanesulfonate and imaged by brightfield microscopy on a Leica M205 FA stereomicroscope.

\section{Genotyping of irf8-/- mutants}

Adult (>30 dpf) zebrafish arising from heterozygous irf8 mutant incrosses were anaesthetised in $40 \mu \mathrm{g} / \mathrm{ml}$ tricaine methanesulfonate and a lobe of caudal fin removed by scalpel. After clipping, fins were digested to extract DNA using $10 \mathrm{mg} / \mathrm{ml}$ Prot $\mathrm{K}$, incubated at $65 \circ \mathrm{C}$ for 1 hour. This incubation ends with 15 minutes at $95^{\circ} \mathrm{C}$ to denature the Proteinase K. A section of irf8 flanking the mutation locus was then amplified from the extracted DNA by PCR using Forward -ACATAAGGCGTAGAGATTGGACG and Reverse -GAAACATAGTGCGGTCCTCATCC primers and REDTaq ${ }^{\circledR}$ ReadyMix ${ }^{\mathrm{TM}}$ PCR Reaction Mix. The PCR product was then digested for 30 minutes at $37^{\circ} \mathrm{C}$ using AVA1 restriction enzyme (New England Bioscience) and the product run on a $2 \%$ agarose gel. WT $=$ Aval digest site is present $=$ PCR product is cleaved to give two bands with sizes of approximately 200 and $100 \mathrm{bp}$. irfo ${ }^{-/-}=$Aval digest site is absent due to mutation $=$ PCR product is not cut. A single band is observed with a size of 286 bp. irf ${ }^{+/-}=$Three bands as above.

Microinjections were performed on larvae at 72 hpf using a Narishige IM-300 Microinjector and pulled thin wall glass capillaries (Harvard Apparatus), administered under anaesthesia by intravenous microinjection through the cardiac sinus venosus (SV) that drains the common cardinal vein (CCV). An injection volume of $1 \mathrm{~nL}$ was used for all intravenous injections to minimise disruption to blood volume. 
668 For propidium iodide intravital staining, $1 \mathrm{~nL} 100 \mu \mathrm{g} / \mathrm{ml}$ propidium iodide in DPBS was 669 injected immediately following injury at $0.5 \mathrm{hpi}$. Larvae were then immediately imaged 670 by heartbeat-synchronised light-sheet microscopy at $1 \mathrm{hpi}$. Injection of recombinant 671 zfIFN- $\gamma$-rel (IFN-1.1) (Kingfisher Bioscience) was administered as a single 1nL 132nM 672 dose at $72 \mathrm{hpf}$. Lyophilised IFN- $\gamma$-rel was reconstituted in PBS $+0.1 \%$ BSA (carrier 673 protein) and PBS $+0.1 \%$ BSA was used as the vehicle control solution. Injections of recombinant zfVEGFaa (Kingfisher Bioscience) were administered as single 1nL 0.25 $\mathrm{ug} / \mathrm{ul}$ doses at $72 \mathrm{hpf}$ (protein reconstituted as above).

\section{Histological staining}

To detect cell death at the injured ventricle, whole-mount larval TUNEL staining was performed. Larvae were fixed in 4\% PFA for 30 minutes and transferred to 1:10 dilution of PBS. Larvae were subsequently digested in $1 \mu \mathrm{g} / \mathrm{ml}$ Proteinase $\mathrm{K}$ for 1 hour. Larvae were re-fixed in 4\% PFA for 20 minutes and subsequently washed in PBT. TUNEL staining was performed using ApopTag Red In situ kit (MilliporeSigma) to label apoptotic cells, as described previously ${ }^{26}$. Stained hearts were imaged using LSFM.

EdU staining was performed by incubating larvae in $1 \mathrm{mM}$ EdU (5-ethynyl-2'deoxyuridine) (Abcam) in $1 \%$ DMSO (Sigma Aldrich) in conditioned water $+0.003 \%$ phenylthiourea (Thermo Fisher Scientific) for 24 hours beginning either at 0 hpi or 24 hpi depending on the experiment. Larvae were incubated at $28.5^{\circ} \mathrm{C}$ in the dark. Larvae were then fixed for 2 hours at room temperature in 4\% PFA, permeabilised in permeabilisation solution (PBS-Triton-X $0.1 \%+1 \%$ Tween $+1 \%$ DMSO) and pericardium punctured using a glass microinjection needle (further improving permeability). Larvae were then washed twice in PBS-3\% BSA and incubated for 2

694 hours at room temperature in CLICK reaction mixture from Click-iT ${ }^{\mathrm{TM}}$ EdU Imaging Kit with Alexa Fluor ${ }^{\mathrm{TM}} 594$ (Invitrogen) made according to manufacturers' instructions. imaged by LSFM.

\section{Heart lesion size quantification}


701 Larval hearts expressing the transgene myl7:GFP were imaged by heartbeatsynchronised light-sheet imaging as described above. Exposure was kept consistent at $10 \mathrm{~ms}$, along with $z$ slice spacing $(1 \mu \mathrm{m})$, and heart contraction phase was locked to mid diastole for all larvae. Z stacks were surface rendered in IMARIS (Bitplane) based on absolute intensity, and software-suggested segmentation and rendering parameters. Lesion area, visualised as a render-free hole in the myocardium, was then traced around manually and lesion area quantified in FIJI (National Institutes of Health $)^{74}$.

\section{Ventricular ejection fraction analysis}

Larval hearts of $T g(m y / 7: G F P)$ larvae were imaged at $80 \mathrm{fps}$ in brightfield using a Leica M205 FA epifluorescence stereomicroscope, to capture when the ventricle was in diastole and systole. The ventricular area in diastole and systole was measured manually in FIJI and ventricular ejection fraction calculated using the formula $100 \mathrm{X}$ [(Diastolic Area - Systolic Area)/Diastolic Area ${ }^{25}$. Ventricular ejection fraction by area was then converted to ejection fraction by volume using the formula 'Ejection fraction by area $\times 2.33=$ Ejection fraction by volume' derived in Supplementary Figure 4. Over the small range of ejection fractions that occur in larval hearts, the relationship can be considered to approximate to a linear one.

\section{Quantification of cell number by image analysis}

To quantify the number of cardiomyocytes in $T g(m y / 7: h 2 b-G F P)$ and $\operatorname{Tg}($ myl7:nlsDsRed) larval hearts, z stacks of hearts acquired by LSFM were imported into FIJI and nuclei counted using the plugin Trackmate. Briefly, key segmentation parameters 'Estimated blob diameter' $=5.5$, 'Threshold' $=0.9$ were taken as a starting point, and optimised manually per experiment until all nuclei are counted successfully. The heart atrium is excluded manually by $x$ coordinate filtering and ventricular cardiomyocytes are then automatically counted by the plug in. in Tg(myl7:h2b-GFP) larval hearts, a custom FIJI macro was written to exclude noncardiomyocyte EdU signal. This is necessary as cardiomyocytes have a much lower 
turnover rate than surrounding cells in the pericardium, endocardium and blood and so represent a minority of EdU+ cells. Briefly, the Bersen segmentation method was used to mask areas of GFP fluorescence per z slice and these masks subsequently applied as a crop Rol to EdU signal in the $641 \mathrm{~nm}$ colour channel of RGB images. Slices were then reassembled and merged into maximum intensity projections, where the $\mathrm{FIJI}^{74}$ Trackmate plugin was used to count both the total number of GFP+ cardiomyocyte nuclei and EdU+ cardiomyocyte nuclei. This quantification then allowed the percentage of EdU+ cardiomycytes to be calculated in an unbiased way per larval heart.

\section{Quantification of notch signalling by image analysis}

In order to objectively identify whether the hearts from $\operatorname{Tg}(T p 1: v e n u s-P E S T)$ larvae possessed venus signal in the endocardium above that of background, and were therefore 'notch+', the following approach was used. Treatment groups were blinded to the analyser, and $z$ stacks opened in FIJI. The automatic brightness and contrast function was used to objectively enhance the signal in the heart, and the clear interface between the granular autofluorescence of the chamber blood and the smooth autofluorescence of the myocardium searched for venus expression. The distinctive morphology and location of endothelium allowed for unambiguous identification of venus+ status.

\section{Pharmacological inhibition of larval signalling}

To inhibit VEGF signalling, larvae were bathed in pan-VEGFR antagonist AV951/Tivozanib (Stratech Scientific) 0-48 hpi. AV951 was dissolved in 0.1\% DMSO in conditioned water $+0.003 \%$ phenylthiourea to make a $10 \mathrm{nM}$ solution, with just $0.1 \%$ DMSO in conditioned water $+0.003 \%$ phenylthiourea becoming the vehicle control. In order to pulse larvae with EdU, the original solution was replaced fresh solution, with the addition of $1 \mathrm{mM}$ EdU at $1 \%$ DMSO.

To inhibit notch signalling, larvae were bathed in gamma secretase inhibitor DAPT (Cambridge Bioscience) 0-48 hpi. DAPT was dissolved in $0.2 \%$ DMSO in conditioned 
water $+0.003 \%$ phenylthiourea to make a $100 \mu \mathrm{M}$ solution, with just $0.2 \%$ DMSO in conditioned water $+0.003 \%$ phenylthiourea becoming the vehicle control. Note, DAPT must be dissolved in DMSO prior to the addition of water to prevent precipitation.

In order to inhibit neuregulin-ERBB signalling, the ErBB2 antagonist AG1478 was used. Larvae were bathed in $1.75 \mu \mathrm{M}$ AG1478 (Cambridge Bioscience) dissolved in $0.25 \%$ DMSO in conditioned water + 0.003\% phenylthiourea over 0-48 hpi.

\section{Extraction of larval hearts and RNA extraction}

Following laser injury at $72 \mathrm{hpf} T g(m y l 7: g a / 4:: G F P ; U A S: m R F P)$ larvae were incubated at $28.5^{\circ} \mathrm{C}$ in conditioned media/water $+0.1 \%$ methylene blue $(\mathrm{w} / \mathrm{v})+0.003 \%$ phenylthiourea. At $48 \mathrm{hpi}$ uninjured and injured larvae were given an overdose of tricaine at $400 \mu \mathrm{g} / \mathrm{ml}$, following which hearts were extracted. We adapted the protocol of Burns and MacRae ${ }^{75}$ to increase the yield of heart retrieval from $\sim 50 \%$ to $\sim 70 \%$. Briefly, $\sim 30$ larvae were placed in $2 \mathrm{~mL}$ eppendorf tubes, the conditioned water drained and replaced with ice cold Leibovitz's L-15 Medium supplemented with 10\% FCS. A 19-gauge needle coupled to a $5 \mathrm{~mL}$ syringe was used to shear the larvae by aspiration and therefore dissociate hearts from the rest of the larva. The lysate was then inspected by epifluorescence microscopy and mRFP+ hearts and collected to be kept on ice. Hearts were then digested at for 10 minutes at $4^{\circ} \mathrm{C}$ in protease solution $(5 \mathrm{mM}$ $\mathrm{CaCl} 2,10 \mathrm{mg} / \mathrm{ml} \mathrm{B}$. Licheniformis protease, $125 \mathrm{U} / \mathrm{mL}$ DNase I in 1x PBS) with occasional aspiration to aid digestion, RNA was then extracted using a RNeasy Plus Micro Kit (Qiagen) following direct lysis with RLT lysis buffer according to manufacturer's instructions. RNA concentration was measured by Qubit and integrity by Bioanalyser. RIN score for all samples ranged between 9.6-10.

\section{RNAseq analysis}

RNA was sequenced by Genewiz, Leipzig, Germany using Illumina NovaSeq, PE $2 \times 150$. Genewiz also used deseq2 package in $\mathrm{R}$ to evaluate sequencing quality, trim reads, map to the Danio rerio genome and generate gene counts/hits. Sequence reads

801 were trimmed using Trimmomatic v.0.36. The trimmed reads were mapped to the 802 Danio rerio GRCz10.89 reference genome available on ENSEMBL using the STAR 
803

804

805

806

807

808

809

810

811

812

813

814

815

816

817

818

819

820

821

822

823

824

825

826

827

828

829

830

831

832

833

834

835

836

aligner v.2.5.2b. Unique gene hit counts were calculated by using featureCounts from the Subread package v.1.5.2. Only unique reads that fell within exon regions were counted. The Wald test was used to generate $p$-values and log2 fold changes. A gene ontology analysis was performed on the statistically significant set of genes by implementing the software GeneSCF v.1.1-p2. The zfin GO list was used to cluster the set of genes based on their biological processes and determine their statistical significance. The volcano plot was generated by a custom $R$ script and heatmap constructed using the pHeatmap package. For the heatmap $z$ scaled log2(Reads) were clustered via Pearson correlation and clusters thresholded based on the resulting dendrogram. The heatmap was generated using the pHeatmap function in R.

\section{Statistics}

Graphs and statistics were curated in GraphPad Prism 9.1 software (GraphPad Software). Data were analysed by student $t$-test, one-way ANOVA or two-way ANOVA followed by an appropriate multiple comparison post hoc test. All statistical tests, $p$ values and $n$ numbers used are given in figure legends, $p<0.05$ was deemed significant in all experiments.

\section{Acknowledgments}

This work was funded by a British Heart Foundation (BHF) CoRE award (RE/13/3/30183), Medical Research Scotland studentship (PhD-1049-2016), NC3R studentship (NC/P002196/1), BHF New Horizons grant (NH/14/2/31074), and a Medical Research Council UK award (MR/K013386/1). Bioinformatics and RNAseq performed by Genewiz, Leipzig, Germany. We also acknowledge Amelia EdmondsonStait, University of Edinburgh, for her advice and input on RNAseq analysis.

\section{Author Contributions}

$F A B$ conceived of and designed the study. $F A B, A K$ and $G M$ carried out all experiments. Image analysis was performed by FB and AK. LSFM-related technical contributions were provided by JMT. FAB wrote the manuscript. KRS, MEMO, EGS and MB provided expertise regarding all RNA work. KRS helped optimise larval heart 
extraction and RNA extraction. JMT, CST, MEMO, JJM, GM, MB, AGR, and MAD

838 edited the manuscript. MAD, AGR, and CST supervised the study. All authors

839 contributed to the article and approved the submitted version.

840

841

Figures

842

a

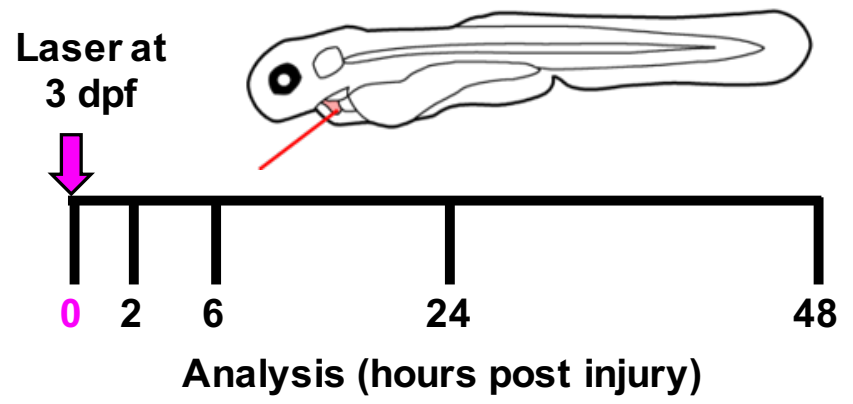

b Tg(mpeg1:GFP;csf1ra:mCherry)
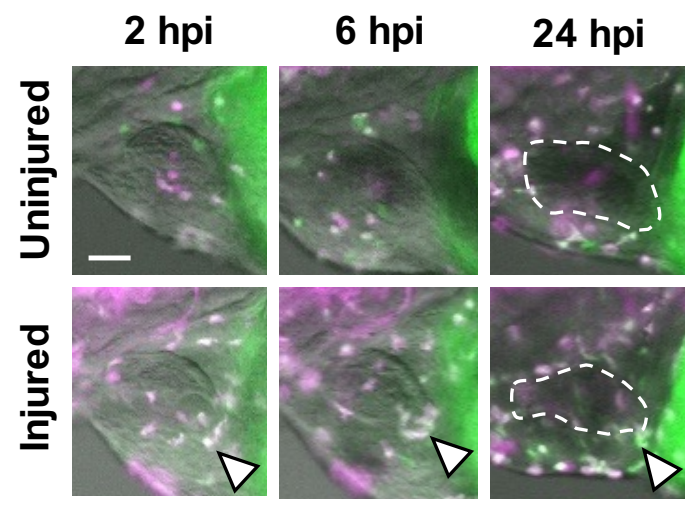

e

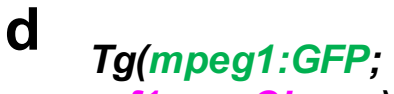
csf1ra:m Cherry)
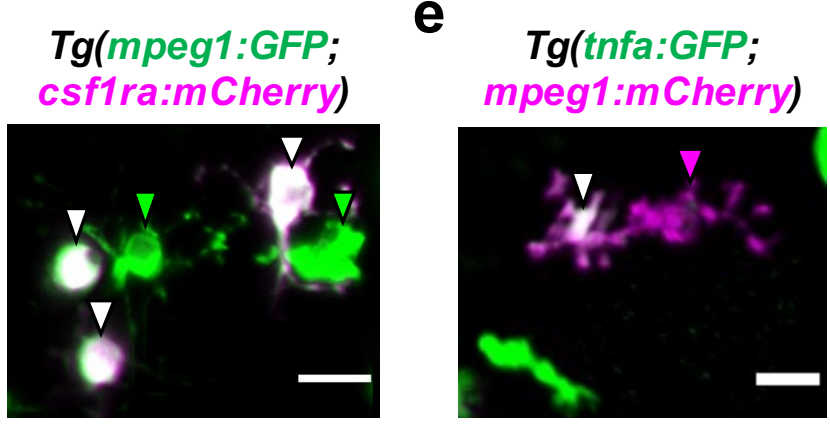

h
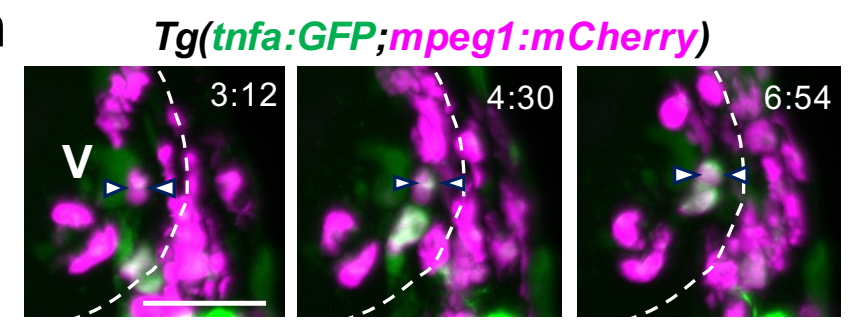

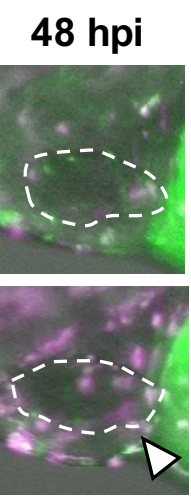

C
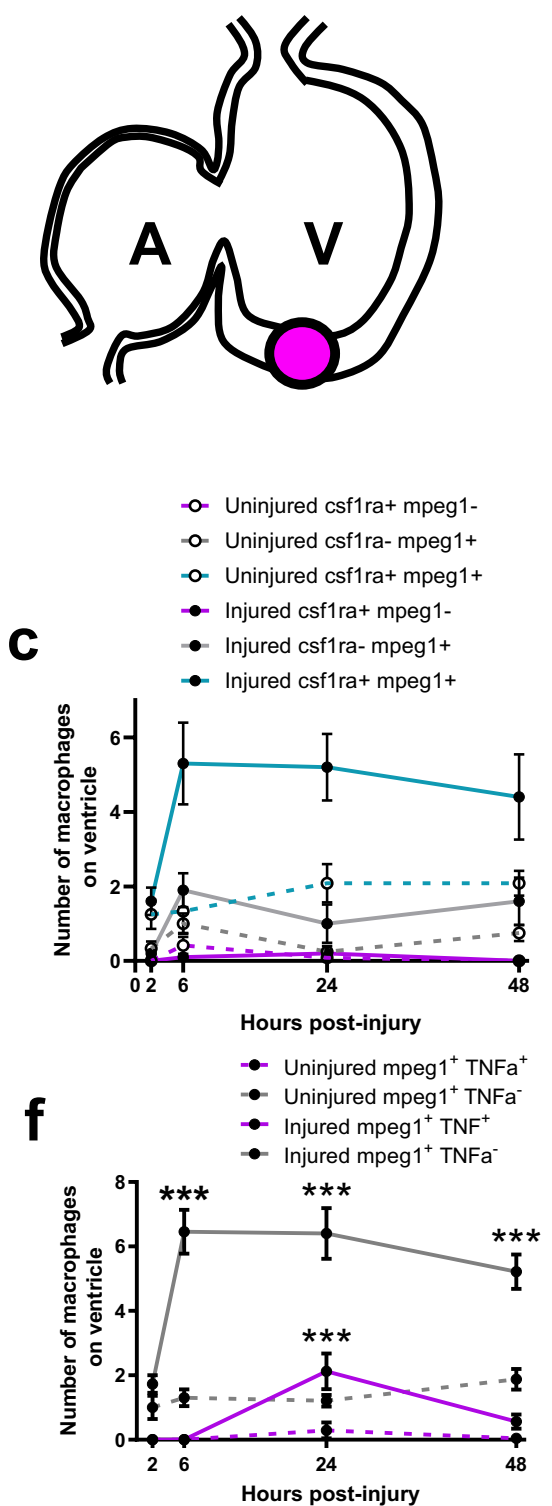

g

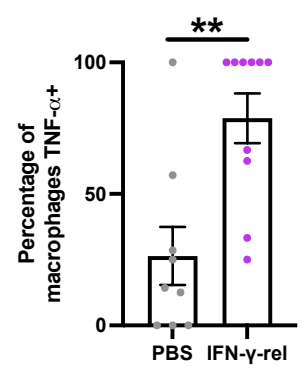


844 Figure 1: Cardiac macrophages display heterogeneity and plasticity following 845 injury.

846 (a) Schematic illustrating the cardiac laser injury model, with imaging timepoints 847 marked (left) and the injury site at ventricular apex of a $3 \mathrm{dpf}$ larval heart marked 848 (magenta circle) (right). (b) Representative lateral view epifluorescence images of 849 uninjured and injured hearts at the standard timepoints in 850 Tg(mpeg1:GFP;csf1ra:gal4:UAS:NfsB-mCherry) (abbreviated to 851 mpeg1:GFP;csf1ra:mCherry in all panels) illustrating macrophage heterogeneity, 852 white arrow = ventricular apex; dashed line = heart outline. (c) Quantification of the 853 number of csf1ra+mpeg1-, csf1ra-mpeg1+ and csf1ra+mpeg1+ macrophages on the 854 ventricle in uninjured and injured larvae at standard timepoints, $n=10-12$. (d) 855 Representative LSFM image of csf1ra-mpeg1+ and csf1ra+mpeg1+ macrophages of 856 different morphologies. (e) Representative LSFM image of tnfa+mpeg1+ and tnfa857 mpeg1+ macrophages. (f) Quantification of number of tnfa+mpeg1+ and tnfa-mpeg1+ 858 macrophages on the ventricle in uninjured and injured larvae at standard timepoints, $859 \mathrm{n}=10-25$. (g) Time-lapse timepoints for injured Tg(tnfa:GFP;mpeg1:mCherry) 860 ventricles imaged live in the larvae by heartbeat-synchronised LSFM microscopy 861 illustrating macrophage plasticity. Timestamps indicated, dashed line $=$ ventricle 862 outline; arrows = macrophage converting to tnfa+. (h) Quantification of the percentage 863 of tnfa + macrophages at 24 hpi following injection with IFN- $\gamma$-rel or PBS, $n=10$. Scale 864 bar $=50 \mu \mathrm{m}(\mathrm{b} \& \mathrm{~h}), 10 \mu \mathrm{m}$ (d \& e). ${ }^{* *} p \leq 0.01,{ }^{* * *} p \leq 0.001$, (c \& f) 2way ANOVA followed 865 by Holm-Sidak's Post-hoc test and (g) ttest.

866 


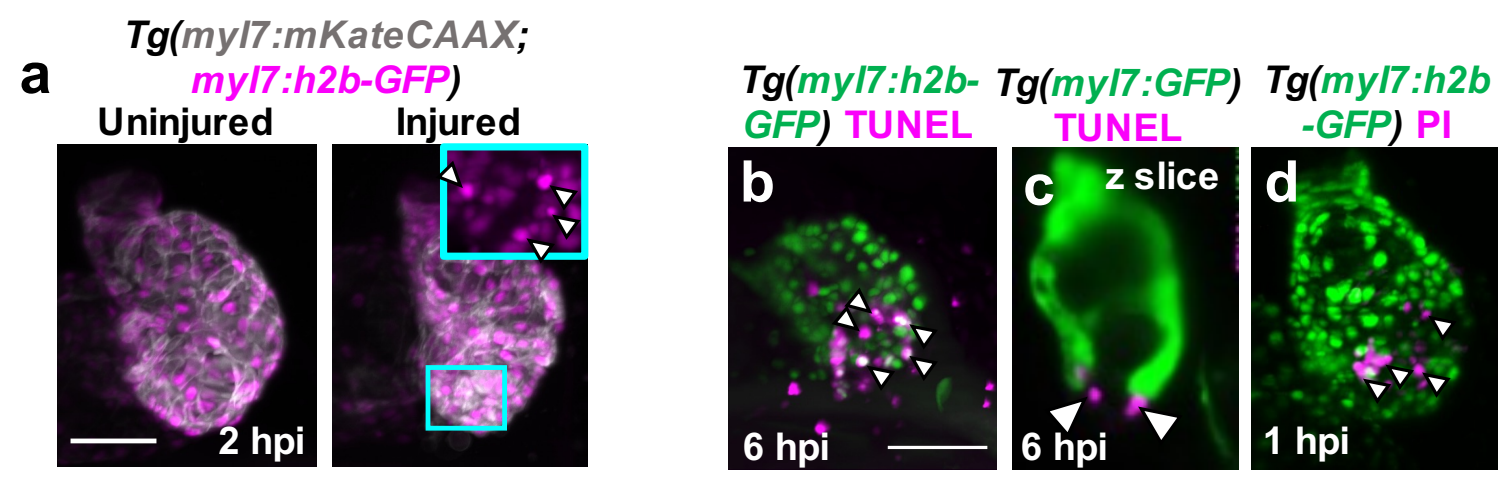

e $T g(m y l 7: h 2 b-G F P)$ TUNEL
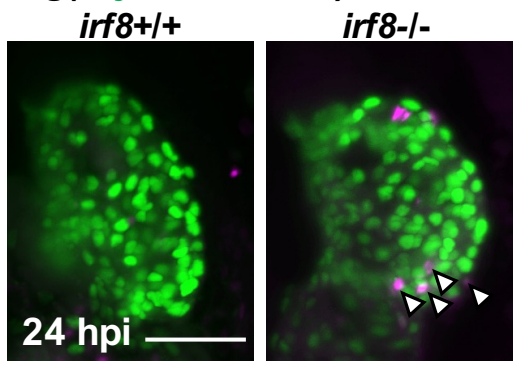

f

Tg(myl7:h2b-GFP) TUNEL
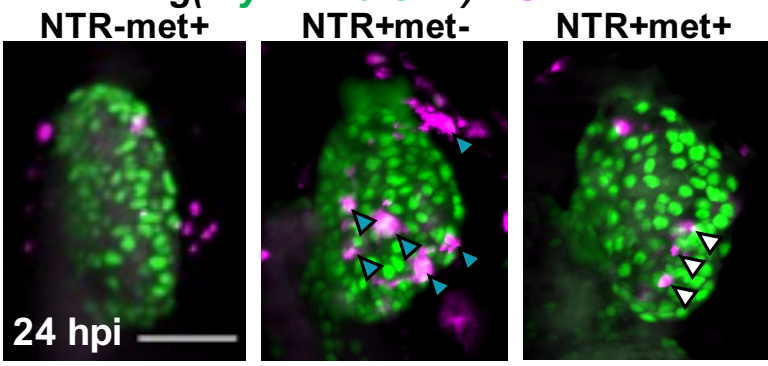

g

h
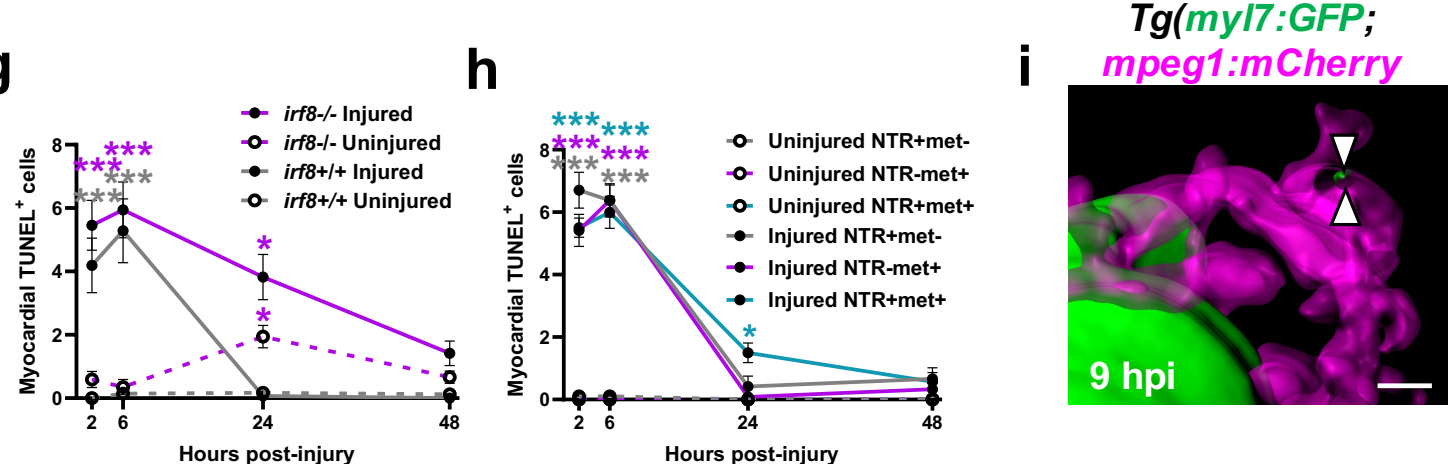

867

868 Figure 2: Macrophages are required for timely removal of apoptotic

869 cardiomyocytes.

870 (a) Representative LSFM images of uninjured and injured $\operatorname{Tg}(\mathrm{my} / 7: \mathrm{h} 2 b$ -

871 GFP;myl7:mKateCAAX) ventricles. Cyan outlined zoom panel highlights condensed

872 nuclei (white arrowheads). Representative LSFM images of TUNEL stained hearts 6

873 hpi in (b) $T g(m y / 7: h 2 b-G F P)$ and (c) $T g$ (myl7:GFP) larvae. White arrowheads =

874 apoptotic cardiomyocytes/myocardium. (d) Representative LSFM image of a

875 propidium iodide $(\mathrm{PI})$ stained $\mathrm{Tg}($ myl7:h2b-GFP) heart at $1 \mathrm{hpi}$. White arrowheads $=$

876 necrotic debris. (e) Representative LSFM images of $i r f 8^{+/+}$and irf8 $8^{-/-} \operatorname{Tg}(m y l 7: h 2 b-G F P)$

877 hearts stained by TUNEL at 24 hpi. White arrowheads = TUNEL+ cells. (f)

878 Representative LSFM images of injured Tg(myl7:h2b-GFP;csfr1a:NfsB-mCherry)

879 ventricles per macrophage ablation model injury group at $24 \mathrm{hpi}$. Cyan arrowheads =

880 Macrophages and white arrowheads $=$ TUNEL + cells. $(g)$ Quantification of TUNEL + 
881 myocardial cells in uninjured and injured, irf8 ${ }^{+/+}$and $i f f 8^{-/-} \mathrm{Tg}(\mathrm{myl7}: \mathrm{h} 2 \mathrm{~b}-\mathrm{GFP})$ ventricles, $882 \mathrm{n}=15-29$. (h) Quantification of TUNEL+ myocardial cells in uninjured and injured $883 \mathrm{Tg}$ (myl7:h2b-GFP;csfr1a:NfsB-mCherry) ventricles per macrophage ablation group, $884 \mathrm{n}=10-12$. (i) Surface render of LSFM-acquired $z$ stack, surfaced-rendered with $50 \%$ 885 transparency at $9 \mathrm{hpi}$, showing internalised myocardial debris (white arrowheads) in a 886 macrophage in a Tg(myl7:GFP;mpeg1:mCherry) larva. Scale bars $=50 \mu \mathrm{m}$ for panels 887 a-f and $20 \mu \mathrm{m}$ for panel (i). All images are 3D LSFM shown as maximum intensity 888 projections unless otherwise stated. ${ }^{*} p \leq 0.05,{ }^{* *} p \leq 0.01,{ }^{* * *} p \leq 0.0012$ way ANOVA 889 followed by Holm-Sidak's Post-hoc tests.

890 


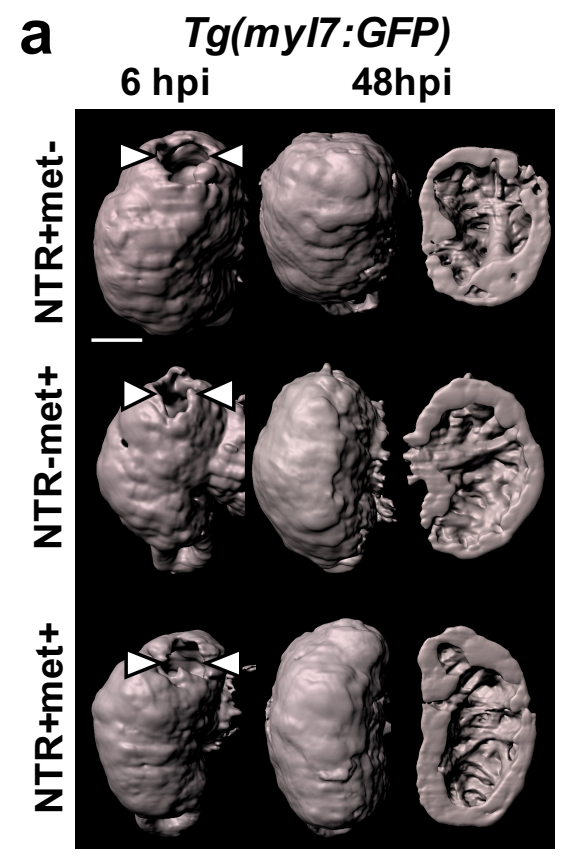

b

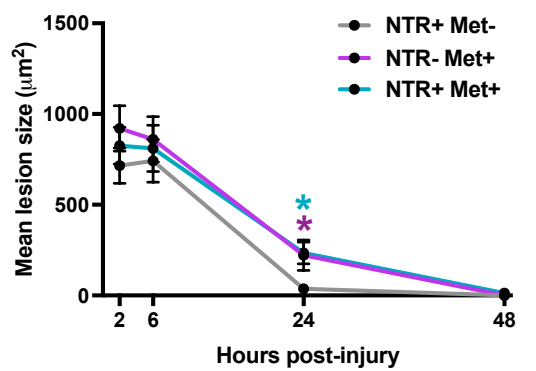

C
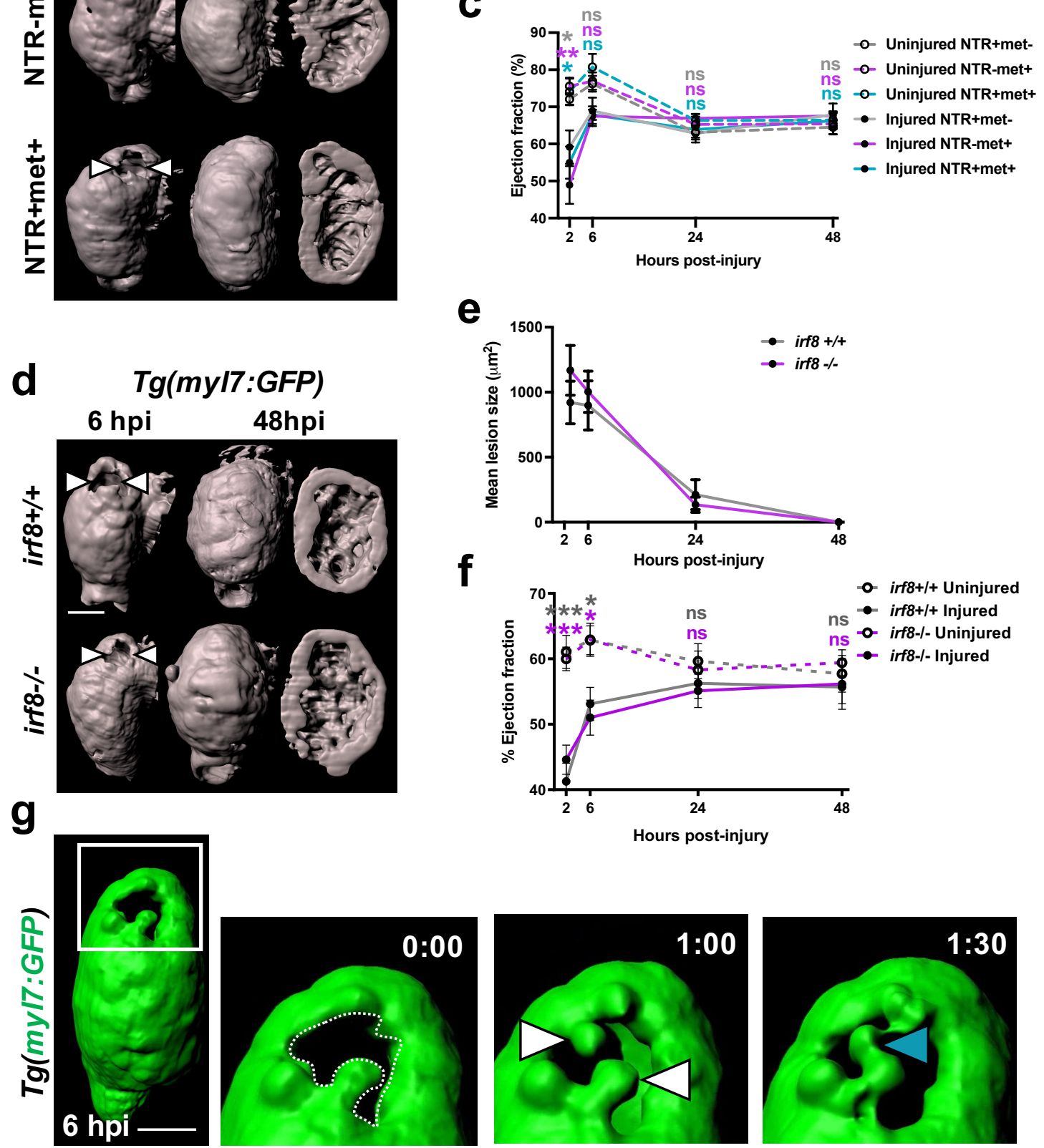

892 Figure 3: Macrophages not required for the recovery of cardiac structure or 893 function.

894 (a) Representative GFP surface-renders of LSFM z-stacks of injured ventricles in 895 Tg(myl7:GFP;csfr1a:NfsB-mCherry) larvae, macrophage ablation groups as indicated 
896 in the figure. Abluminal myocardial surface is shown at $6 \mathrm{hpi}$ (left) and abluminal and

897 luminal surfaces shown at $48 \mathrm{hpi}$ following regeneration (middle \& right). White 898 arrowheads = laser lesion. (b) Quantification of mean lesion size in injured $899 T g$ (myl7:GFP;csfr1a:NfsB-mCherry) larvae per macrophage ablation group, $\mathrm{n}=11-22$. 900 (c) Quantification of ventricular ejection fraction in uninjured and injured 901 Tg (myl7:GFP;csfr1a:NfsB-mCherry) larvae per macrophage ablation group, $\mathrm{n}=10-12$. 902 (d) Representative GFP surface-renders of light-sheet-acquired z-stacks of injured 903 ventricles from irf8 ${ }^{+/+}$and irf8 ${ }^{-/} \operatorname{Tg}(m y / 7: G F P)$ larvae. Abluminal myocardial surface is 904 shown at $6 \mathrm{hpi}$ (left) and abluminal and luminal surfaces shown at $48 \mathrm{hpi}$ following 905 regeneration (middle \& right). White arrowheads = laser lesion. (e) Quantification of 906 mean lesion size in injured $\mathrm{irf8}^{+/+}$and irf8 ${ }^{-/} \operatorname{Tg}($ myl7:GFP) larvae, $\mathrm{n}=15$. (f) 907 Quantification of ventricular ejection fraction in uninjured and injured irf $8^{+/ /}$and irf $8^{-1-}$ $908 T g(m y / 7: G F P)$ larvae $\mathrm{n}=15-20$. (g) Time-lapse timepoints of a GFP-surface-rendered, 909 injured $T g(m y / 7: G F P)$ ventricle from 6 hpi. White box = zoom panel; white arrowheads $910=$ myocardial buds, cyan arrowhead = myocardial bridge. ${ }^{*} p \leq 0.05,{ }^{* * *} p \leq 0.0012$ way 911 ANOVA followed by Holm-Sidak's Post-hoc tests. Scale bars $=50 \mu \mathrm{m}$ 
a

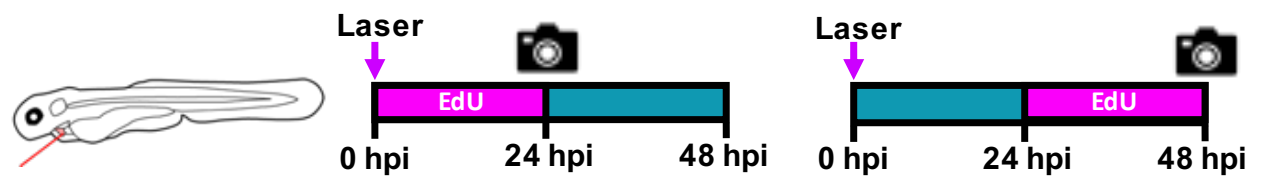

b Tg(myl7:h2b-GFP) EdU Pulse 24-48 hpi Uninjured

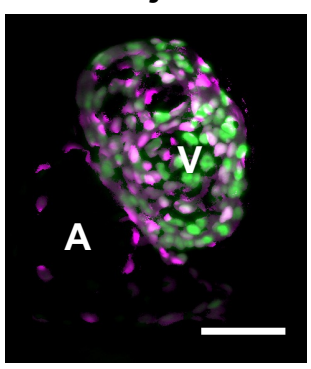
Injured
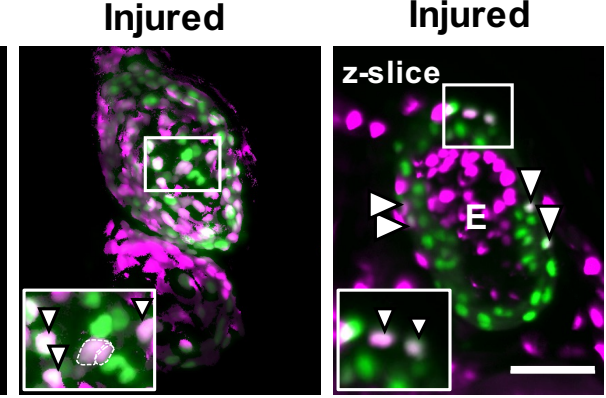

d

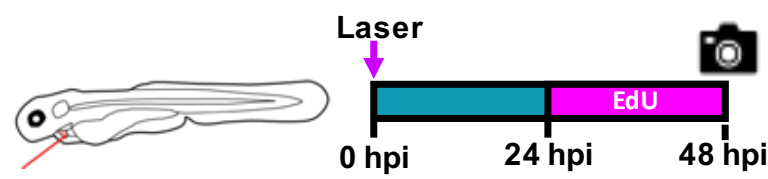

to

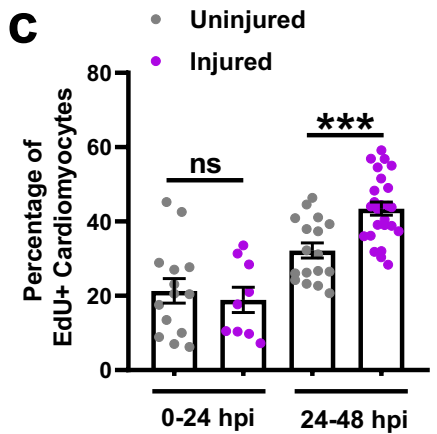

e
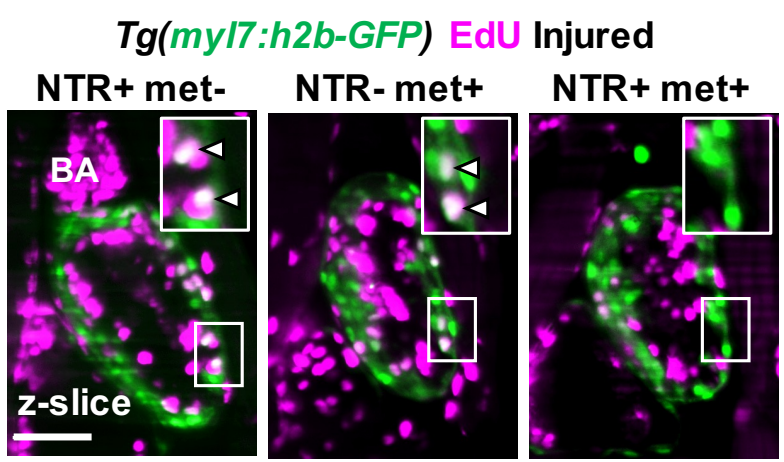

f

- Uninjured

- Injured
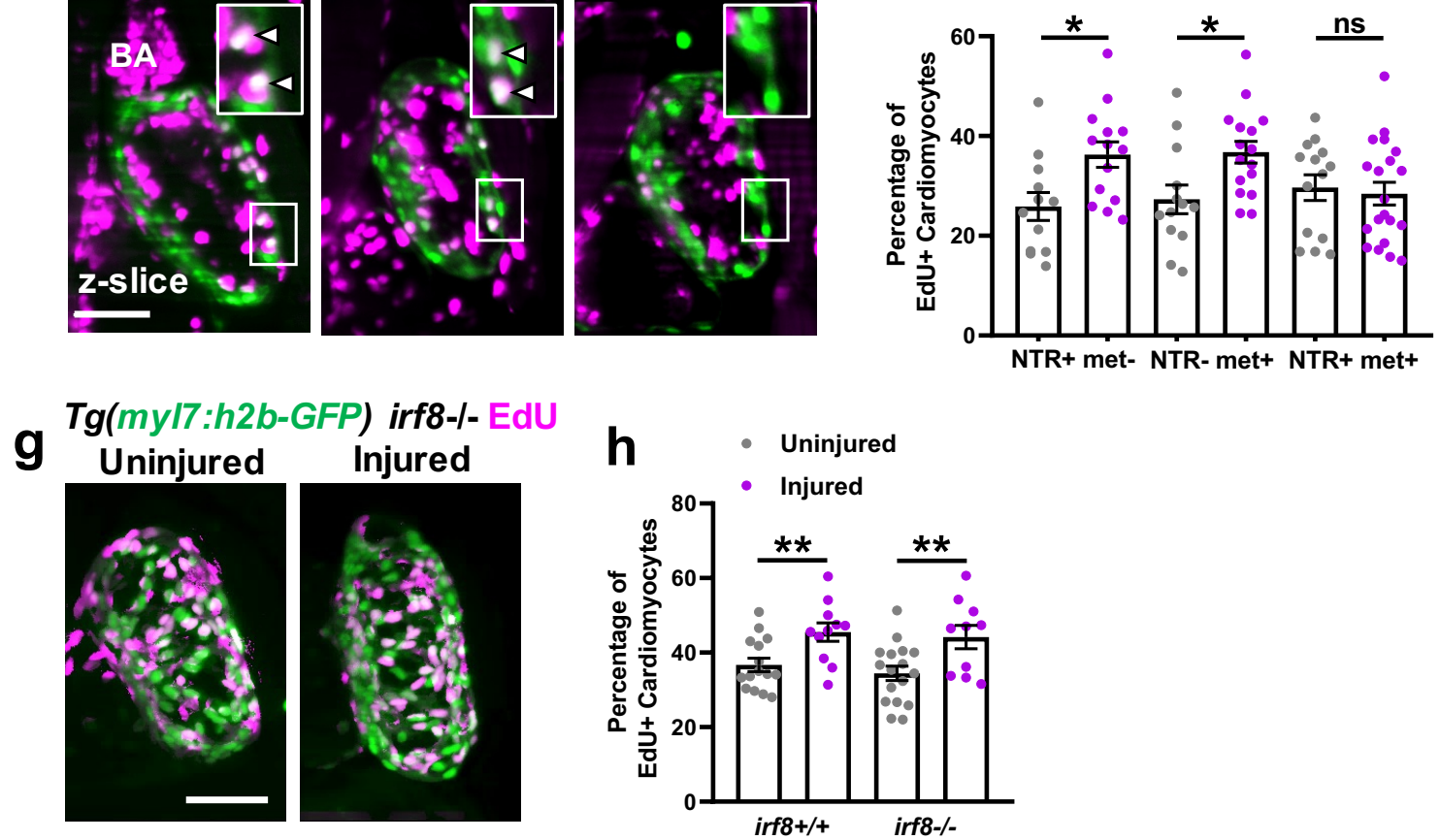

914 Figure 4: Macrophage ablation abolishes injury-dependent cardiomyocyte 915 proliferation.

916 (a) Schematic illustrating EdU pulse strategy for labelling proliferating cardiomyocytes

917 over 0-24 hpi (left) and 24-48 hpi (right). (b) Representative images of EdU-stained

918 hearts from $\operatorname{Tg}(m y / 7: h 2 b-G F P)$ at 48 hpi. Non-myocardial EdU signal is excluded post-

919 acquisition to allow interpretable maximal intensity projections (MIPs). A = atrium, v = 
920 ventricle; white boxes $=$ zoom panels; white arrowheads $=$ EdU + cardiomyocyte nuclei

921 and dashed line = outline of dividing cardiomyocyte daughter nuclei. (c) Quantification

922 of the percentage of ventricular EdU + cardiomyocytes in uninjured and injured

$923 T$ T (myl7:h2b-GFP) hearts pulsed over 0-24 hpi or $24-48$ hpi. ${ }^{* * *} p \leq 0.001$ unpaired $t$

924 test. (d) Schematic illustrating EdU pulse strategy for labelling proliferating

925 cardiomyocytes over 24-48 hpi in Tg(myl7:h2b-GFP;csfr1a:NfsB-mCherry) larvae per

926 standard macrophage ablation groups. (e) Representative images of EdU-stained

927 hearts from $\mathrm{Tg}$ (myl7:h2b-GFP;csfr1a:NfsB-mCherry) acquired by light-sheet

928 microscopy at $48 \mathrm{hpi}$. White boxes $=$ zoom panels; white arrowheads $=\mathrm{EdU}+$

929 cardiomyocyte nuclei and BA = bulbous arteriosus. (f) Quantification of the percentage

930 of ventricular EdU+ cardiomyocytes in uninjured and injured $\mathrm{Tg}$ (myl7:h2b-

931 GFP;csfr1a:NfsB-mCherry) hearts pulsed over $24-48$ hpi. ${ }^{*} p \leq 0.05$ Kruskal-Wallis test

932 and Dunn's multiple comparison post-hoc test. (g) Representative images of uninjured

933 and injured EdU-stained hearts from irf8 ${ }^{-/} \mathrm{Tg}$ (myl7:h2b-GFP) acquired by light-sheet

934 microscopy at $48 \mathrm{hpi}$. Non-myocardial EdU signal is excluded post-acquisition to allow

935 interpretable maximal intensity projections. (h) Quantification of the percentage of

936 ventricular EdU+ cardiomyocytes in uninjured and injured irf8 ${ }^{+/+}$and irf8 ${ }^{-/}$

$937 \mathrm{Tg}($ myl7:h2b-GFP) hearts pulsed 24-48 hpi. All images are maximum intensity

938 projections of 3D LSFM stacks, unless otherwise stated. Scale bars $=50 \mu \mathrm{m} .{ }^{* *} p \leq 0.01$

939 unpaired t test

940 


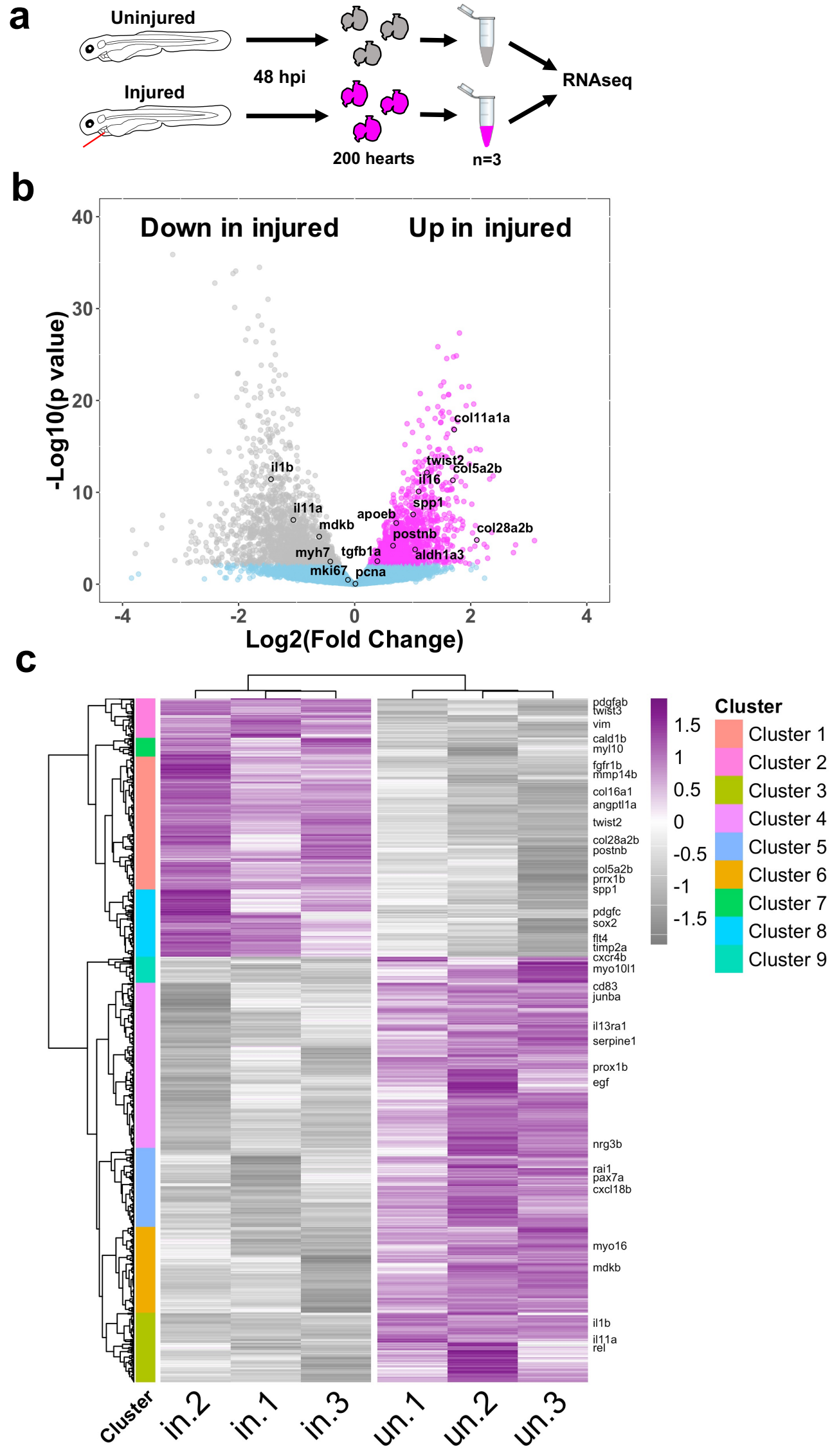


943 (a) Schematic illustrating the extraction of uninjured and injured hearts at $48 \mathrm{hpi}$ and 944 the pooling of 200 hearts per biological replicate for RNAseq, $n=3$. (b) Volcano plot 945 showing the $\log _{2}$ (Fold Change) and - $\log _{10}(\mathrm{p}$ value) for transcripts of each detected 946 gene. Genes whose adjusted $p$ values fall below 0.05 are deemed statistically non947 significant and coloured blue. Genes up regulated in injured hearts are coloured 948 magenta and those upregulated in uninjured hearts are coloured grey. (c) Heatmap 949 displaying statistically significantly differentially expressed genes with a $\log _{2}$ (Fold 950 Change) $>0.5$. Genes were hierarchically clustered by Pearson correlation with $z$ 951 scaling. Clusters are indicated on the left with their dendrogram. Magenta = high 952 expression; grey = low expression. Genes with relevance to cardiac regeneration are 953 highlighted as annotations on the right of the plot. $n=3$ 
a

Tg(myl7:mKateCAAX;vegfaa:GFP)

b
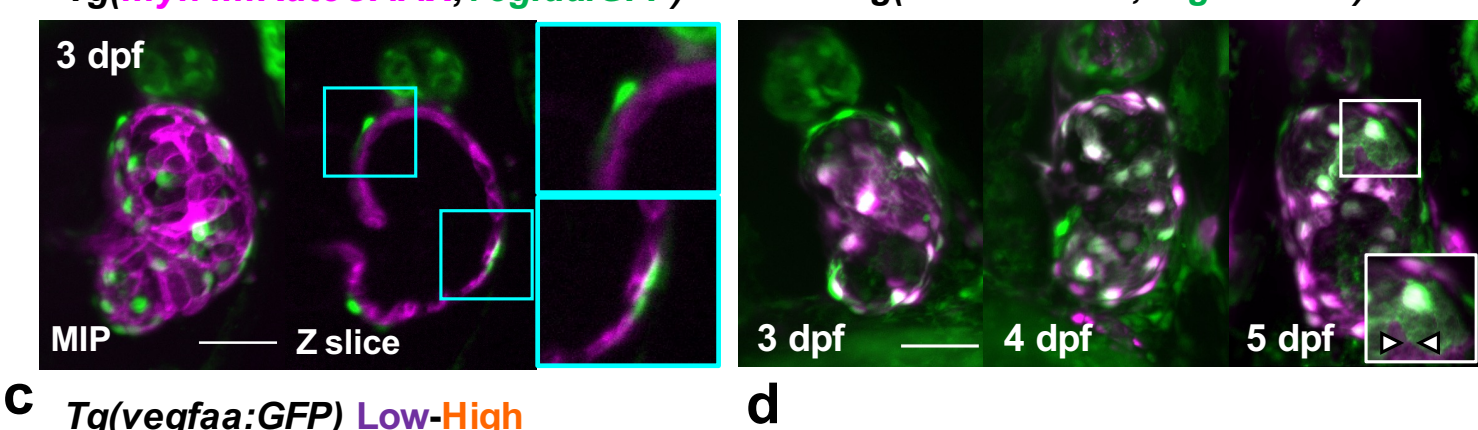

d

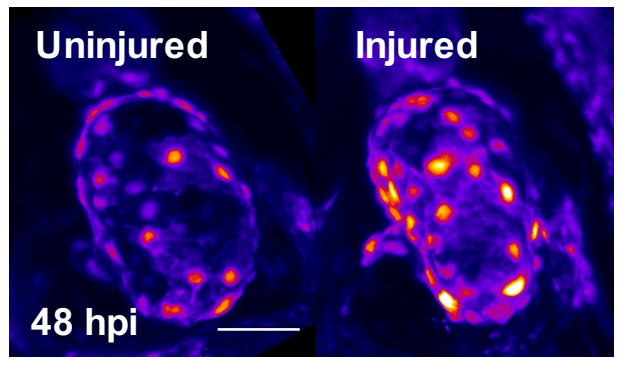

e Tg(vegfaa:GFP; csf1ra:NfsBmCherry;kdrl:mCherry)

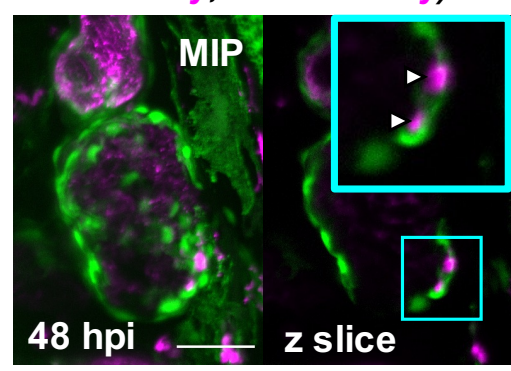

f

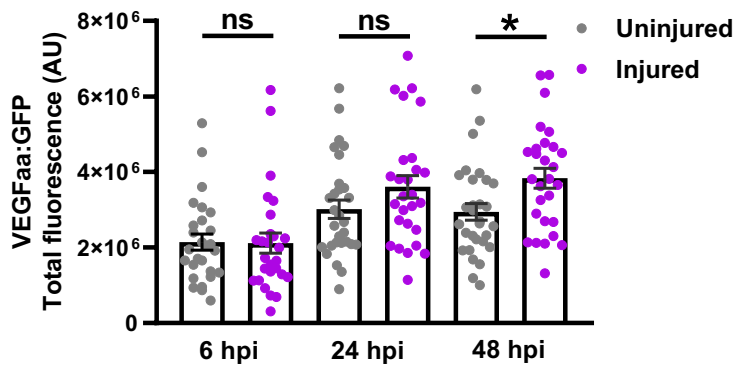

Tg(vegfaa:GFP) Low-High NTR-met+ NTR-met+ NTR+met- NTR+met+

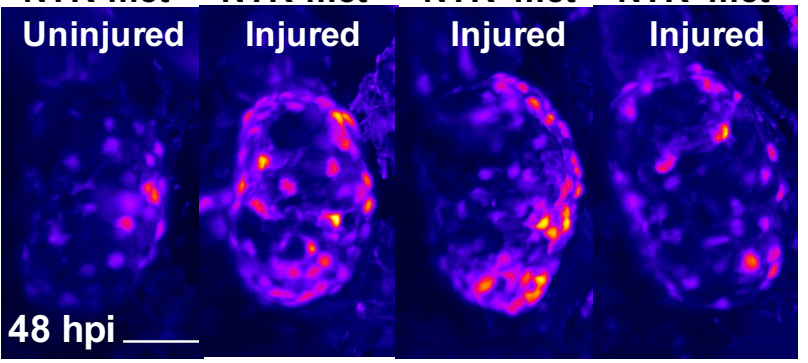

g
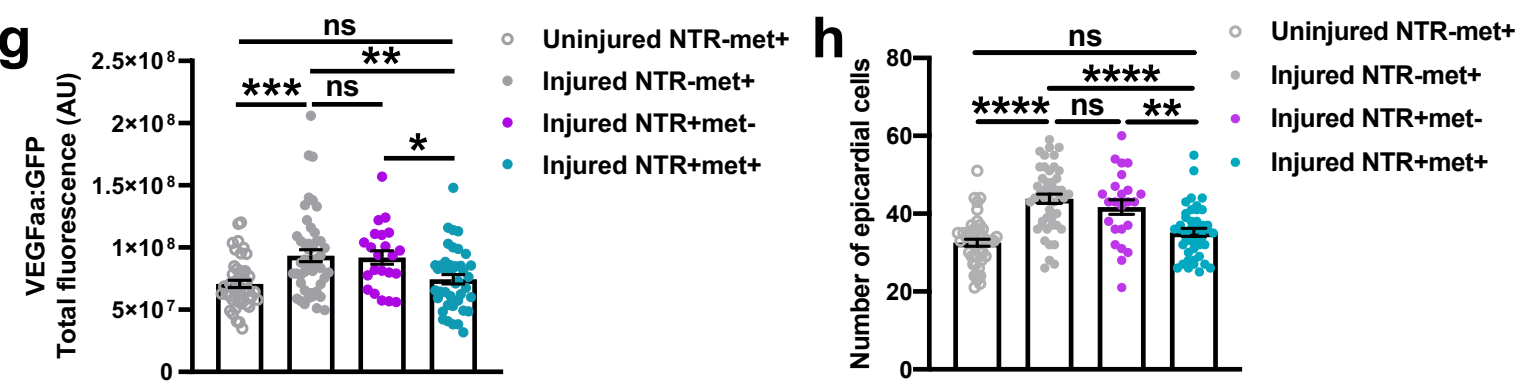

956 Figure 6: Macrophages stimulate epicardial cell number expansion following 957 cardiac injury.

958 (a) Representative LSFM image of an uninjured $3 \mathrm{dpf}$ ventricle from a

959 Tg(myl7:mKateCAAX;myl7:h2b-GFP) larva showing vegfaa+ cells (green) overlying

960 myocardium (magenta). Cyan box = zoom panel. (b) Representative images of 3, 4

961 and $5 \mathrm{dpf}$ ventricles from a Tg(tcf21:DsRed;vegfaa:GFP) larvae acquired by LSFM,

962 showing high colocalization of vegfaa with epicardial marker tcf21. White arrowheads

963 = heterogenous marker expression and white box $=$ zoom panel. (c) Representative 
964 images of uninjured and injured ventricles from $\operatorname{Tg}$ (vegfaa:GFP) larvae acquired at 48

965 hpi by LSFM. "Heat" LUT applied to highlight increased intensity of epicardial 966 vegfaa:GFP in injured hearts. (d) Quantification of total ventricular VEGFaa:GFP

967 fluorescence in uninjured and injured hearts over standard injury model timepoints, $968 n=28-30 .{ }^{*} p \leq 0.05$ One way ANOVA followed by Holms-Sidak's multiple comparison 969 Post-hoc tests. (e) Representative image of a ventricle from a 970 Tg(vegfaa:GFP;csfr1a:NfsB-mCherry;kdrl:hsa.HRAS-mCherry) (abbreviated to $971 \mathrm{kdrl}: \mathrm{mCherry}$ ) larva at $48 \mathrm{hpi}$ showing macrophages in the epicardial-myocardial niche 972 (white arrowheads). Cyan box = zoom panel. (f) Representative LSFM images of 973 uninjured and injured ventricles from Tg(vegfaa:GFP;csfr1a:NfsB-mCherry) larvae 974 from metronidazole-nitroreductase macrophage ablation groups at $48 \mathrm{hpi}$. "Heat" LUT 975 is applied to highlight increase in overall fluorescence in injured groups except 976 NTR+met+. (g) Quantification of total vegfaa:GFP fluorescence $(\mathrm{g})$ and epicardial cell 977 number (h) in uninjured and injured ventricles from Tg(vegfaa:GFP;csfr1a:NfsB978 mCherry) larvae from metronidazole-nitroreductase macrophage ablation groups at $97948 \mathrm{hpi}$. All images are maximum intensity projections of 3D LSFM stacks. Scale bars $980=50 \mu \mathrm{m}, \mathrm{n}=46 .{ }^{*} p \leq 0.05,{ }^{* *} p \leq 0.01,{ }^{* * *} p \leq 0.001$ One way ANOVA followed by Holms981 Sidak's multiple comparison Post-hoc tests.

982 

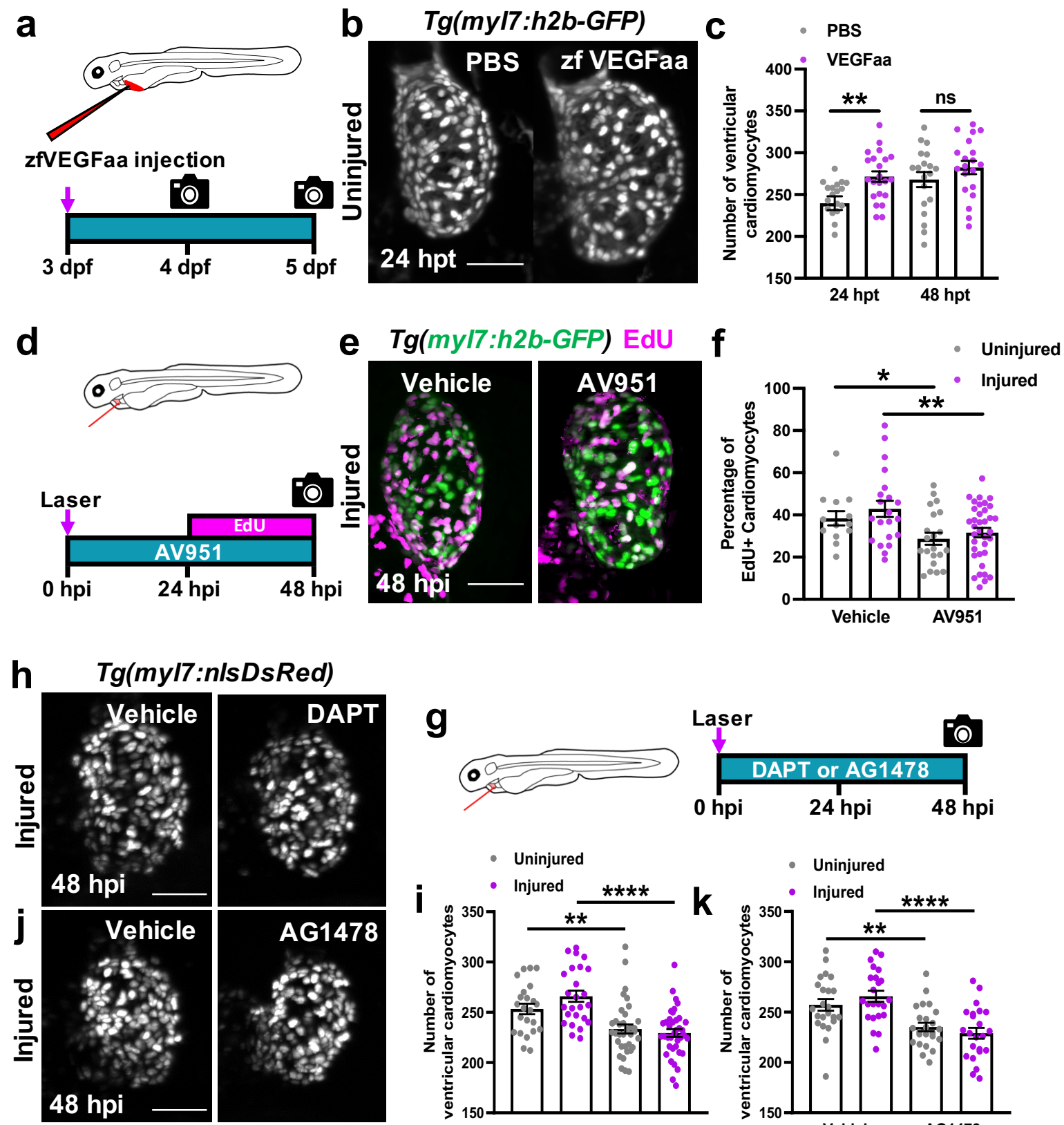

984
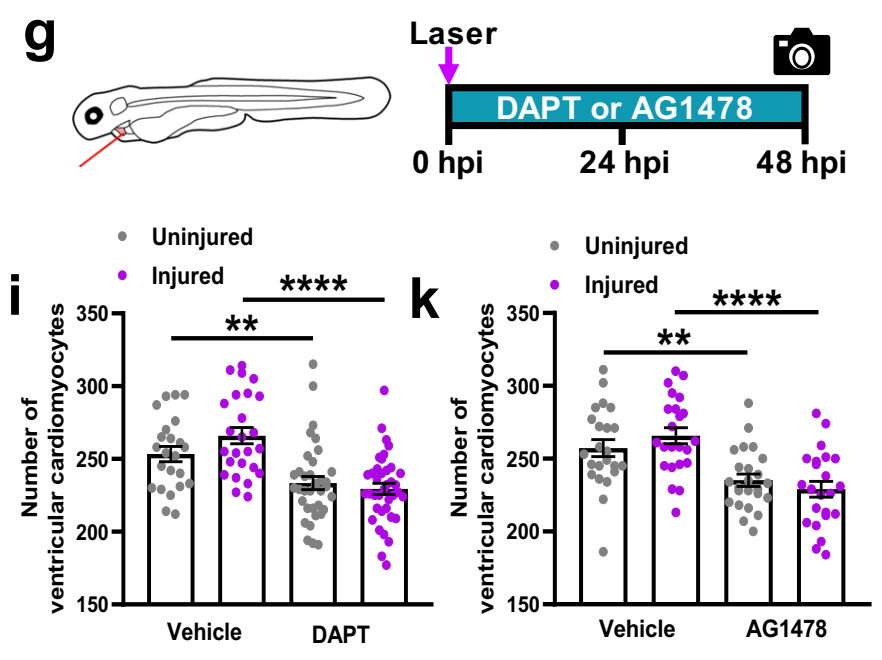

985 Figure 7: VEGFaa signalling is both sufficient and necessary to drive 986 cardiomyocyte proliferation.

987 (a) Schematic illustrating zfVEGFaa treatment strategy via microinjection into the 988 common cardinal vein of uninjured larvae at 72 hpi. (b) Representative LSFM images 989 of $\operatorname{Tg}(m y / 7: h 2 b-G F P)$ larvae at 24 hpi treated with PBS $0.1 \%$ BSA or zfVEGFaa $0.1 \%$ 990 BSA injection. (c) Quantification of ventricular cardiomyocyte number in $\operatorname{Tg}(\mathrm{my} / 7: \mathrm{h} 2 b$ -

991 GFP) larvae at 24 and 48 hpi treated with PBS $0.1 \%$ BSA or zfVEGFaa $0.1 \%$ BSA 992 injection, $\mathrm{n}=20$. ${ }^{* *} p \leq 0.01$ unpaired t test. (d) Schematic illustrating AV951 treatment 993 and EdU pulsing strategy for uninjured and injured larvae. (e) Representative images 994 of injured ventricles from $\operatorname{Tg}($ myl7:h2b-GFP) larvae, EdU stained and bathed in vehicle 
995 or AV951, imaged at 48 hpi by LSFM. Non-myocardial EdU signal is excluded post996 acquisition to allow interpretable maximal intensity projections (MIPs). (f)

997 Quantification of the percentage of EdU+ cardiomyocyte nuclei from uninjured and 998 injured ventricles from $T g(m y / 7: h 2 b-G F P)$ larvae, EdU stained and bathed in vehicle 999 or AV951, $n=13-36 .{ }^{*} p \leq 0.05,{ }^{* *} p \leq 0.01$ unpaired $t$ test. (g) Schematic illustrating the 1000 treatment strategy for DAPT and AV951 bathing of uninjured and injured larvae. (h) 1001 Representative images of injured $T g(m y / 7: n / s D s R e d)$ larvae treated with vehicle or 1002 DAPT, acquired at $48 \mathrm{hpi}$ by LSFM. (i) Quantification of ventricular cardiomyocyte 1003 number in uninjured and injured $T g$ (myl7:h2b-GFP) larvae at 48 hpi treated with 1004 vehicle or DAPT, $n=24-40 .{ }^{* *} p \leq 0.01,{ }^{* * *} p \leq 0.0001$ Unpaired $t$ test.(j) Representative 1005 images of injured $\operatorname{Tg}($ myl7:nlsDsRed) larvae treated with vehicle or AG1478, acquired 1006 at 48 hpi by LSFM. (k) Quantification of ventricular cardiomyocyte number in uninjured 1007 and injured $\operatorname{Tg}(m y / 7: h 2 b-G F P)$ larvae at $48 \mathrm{hpi}$ treated with vehicle or AG1478, $\mathrm{n}=24$. 1008 All images are maximum intensity projections of 3D LSFM stacks. ${ }^{* *} p \leq 0.01$, $1009{ }^{* * * *} p \leq 0.0001$ Unpaired t test. Scale bars $=50 \mu \mathrm{m}$. 


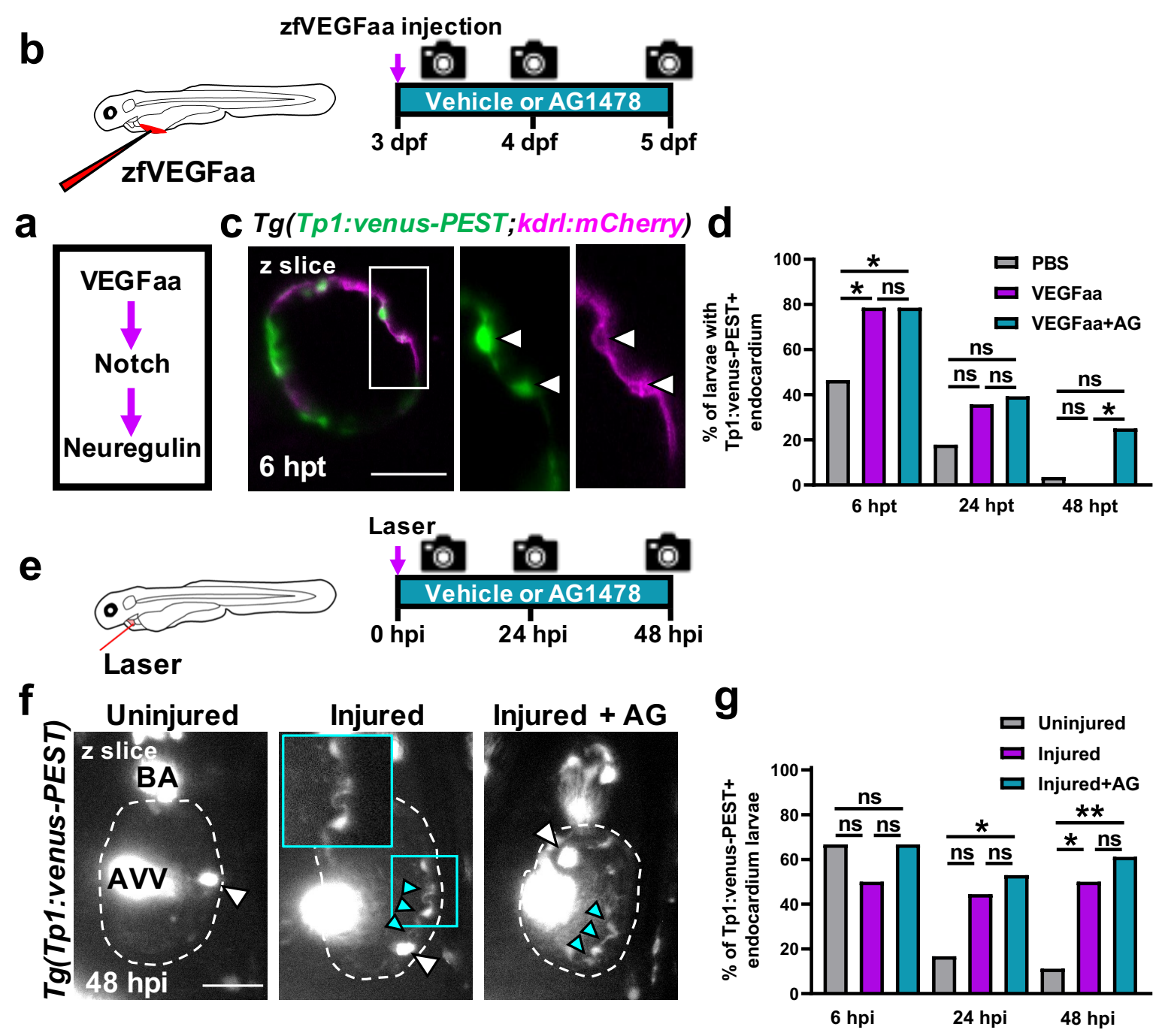

1010

1011 Figure 8: VEGFaa drives cardiomyocyte proliferation by endocardial notch 1012 signalling.

1013 (a) Hypothesised signalling pathway active in uninjured and injured larval hearts

1014 driving cardiomyocyte proliferation. (b) Schematic illustrating the treatment strategy for

1015 the injection of uninjured larvae with zfVEGFaa and continuous bathing in AG1478

1016 solution. (c) Representative LSFM-acquired z plane showing notch expression

1017 colocalising with endocardium in Tg(Tp1:venus-PEST;kdrl:hsa.HRAS-mCherry),

1018 abbreviated in the figure to Tg(Tp1:venus-PEST;kdrl:mCherry). AG1478 abbreviated

1019 to AG, white box = zoom panel. (d) Quantification of the proportion of larvae with

1020 notch+ endocardium at 6, 24, and $48 \mathrm{hpt}$ following zfVEGFaa injection and bathing in

1021 AG1478, $n=28 .{ }^{*} p \leq 0.05$ Fisher's exact test. (e) Schematic illustrating the treatment

1022 strategy for the lasering of larvae and continuous bathing in AG1478 solution. (f)

1023 Representative z plane images of uninjured, injured and injured AG-treated ventricles

1024 from $T g$ (tp1:venus-PEST) larvae acquired by light-sheet microscopy at $48 \mathrm{hpi}$. BA = 
1025 bulbous arteriosus; $\mathrm{AVV}=$ atrioventricular valve; white arrowheads =laterally inhibited 1026 cardiomyocytes, cyan arrowheads $=$ notch + endocardium; cyan box $=$ zoom panel. $(\mathrm{g})$ 1027 Quantification of the proportion of larvae with notch+endocardium at 6, 24, and $48 \mathrm{hpt}$ 1028 following laser injury and bathing in $A G 1478, n=18$. All images are maximum intensity 1029 projections of 3D LSFM stacks unless otherwise stated. ${ }^{*} p \leq 0.05,{ }^{* *} p \leq 0.01$ Fisher's 1030 exact test. Scale bars $=50 \mu \mathrm{m}$.

1031

1032 Supplementary figures

1033 


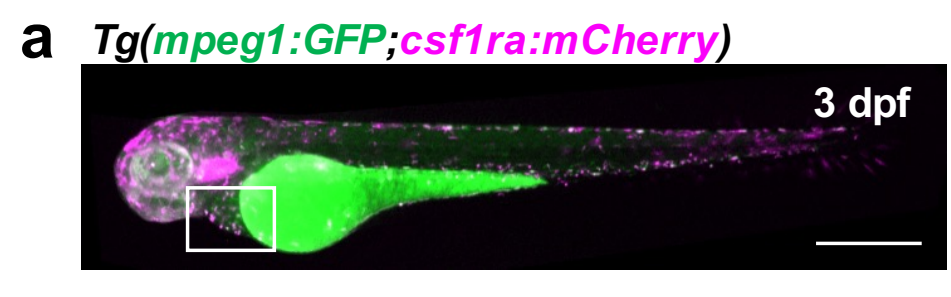

b

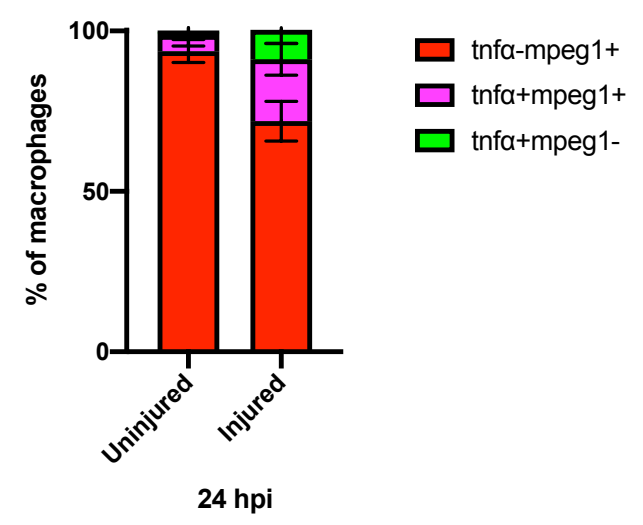

C
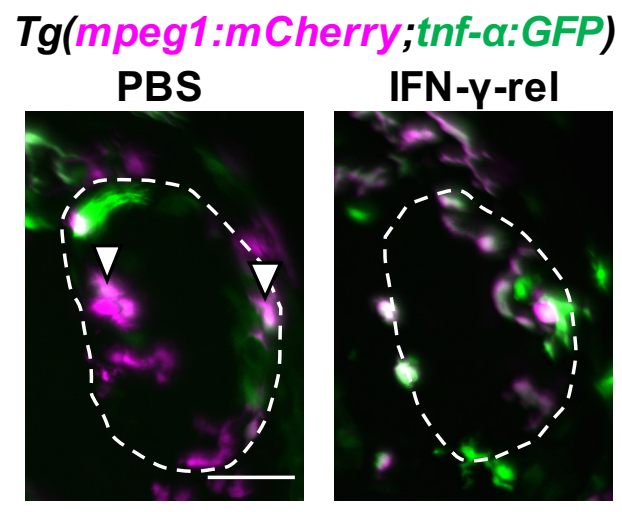

\section{IFN-y-rel}

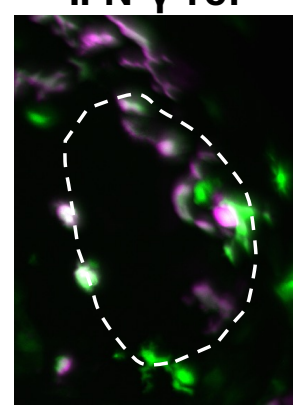

d

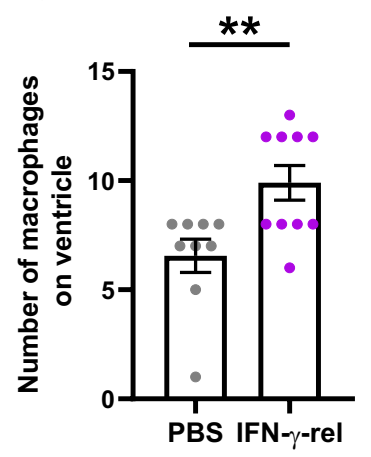

e

Tg(myl7:GFP;mpeg1:GFP) Touching Heart

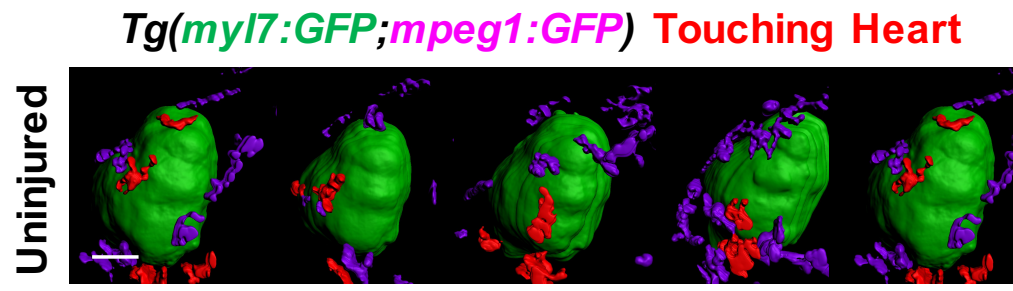

1035 Supplementary figure 1: Cardiac macrophage phenotype in larval zebrafish is 1036 plastic and can be polarised to tnfa+ by IFN- $\gamma$-rel.

1037 (a) Representative epifluorescence image of a $3 \mathrm{dpf}$ Tg(mpeg1:GFP;csf1ra:NfsB-

1038 mCherry) abbreviated to Tg(mpeg1:GFP;csf1ra:mCherry) in the figure, showing an 
1039 anterior-posterior polarity in macrophage expression of csf1ra (higher proportion of 1040 anterior macrophages were csf1ra+). White box $=$ indicated pericardial area. Scale bar

$1041=500 \mu \mathrm{m}$. (b) Quantification of the proportion of macrophages that are tnfa-mpeg1+, 1042 tnfa+mpeg1+ and tnfa+mpeg1- on hearts in uninjured and injured larvae at 24 hpi. (c) 1043 Representative images of hearts from $\operatorname{Tg}$ (mpeg1:mCherry;tnfa:GFP) larvae at 24 hpi 1044 injected with PBS or IFN- $\gamma$-rel. White dashed line = outline of the ventricle; and white 1045 arrowheads $=$ tnfa + mpeg1 + macrophages. Scale bar $=50 \mu \mathrm{m}$. (d) Quantification of the 1046 number of macrophages on the injured ventricle at $24 \mathrm{hpi}$ after injection at 0 hpi with 1047 PBS or IFN- $\gamma$-rel. (e) Time-lapse timepoints of Tg(myl7:GFP;mpeg1:mCherry) hearts 1048 acquired by heartbeat-synchronised LSFM, surface rendered and colour-coded to 1049 show myocardium in green, macrophages on the heart in red and macrophages 1050 elsewhere in purple. Macrophages can be seen to change from stellate to rounded 1051 over time following injury. Scale bar $=50 \mu \mathrm{m}$. 


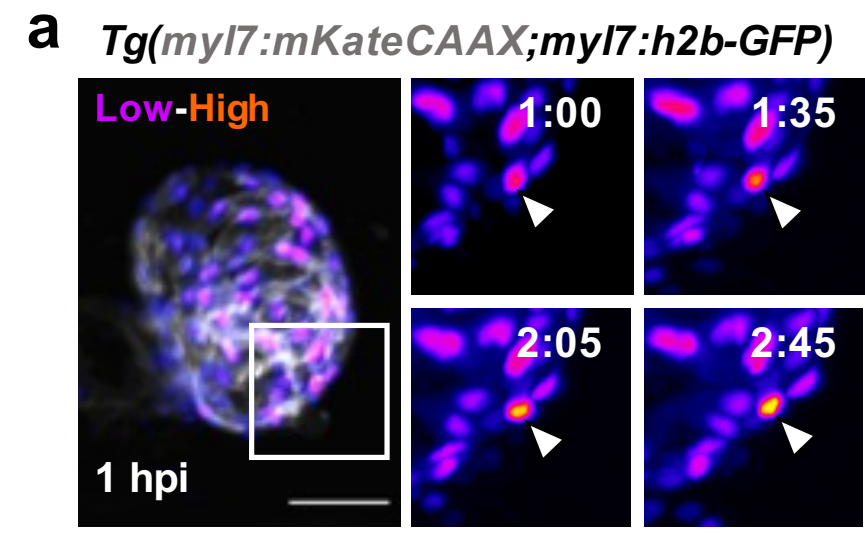

b $\quad \operatorname{Tg}($ mpeg1:GFP; myl7:h2b-GFP; myl7:mKateCAAX) PI

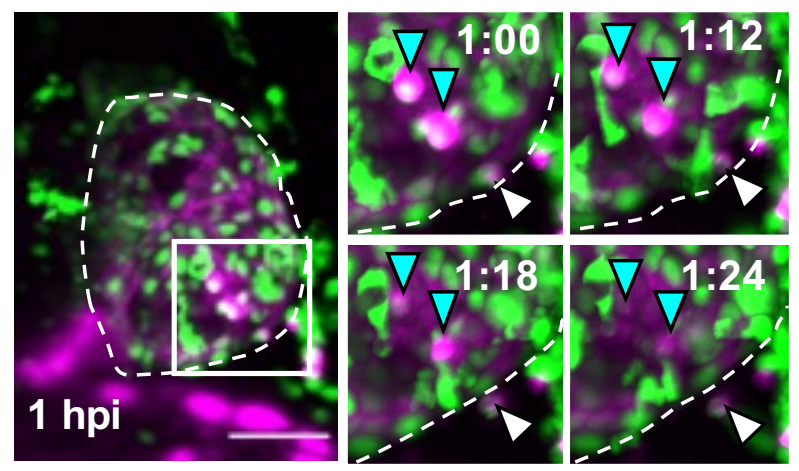

C Tg(myl7:GFP;mpeg1:mCherry)

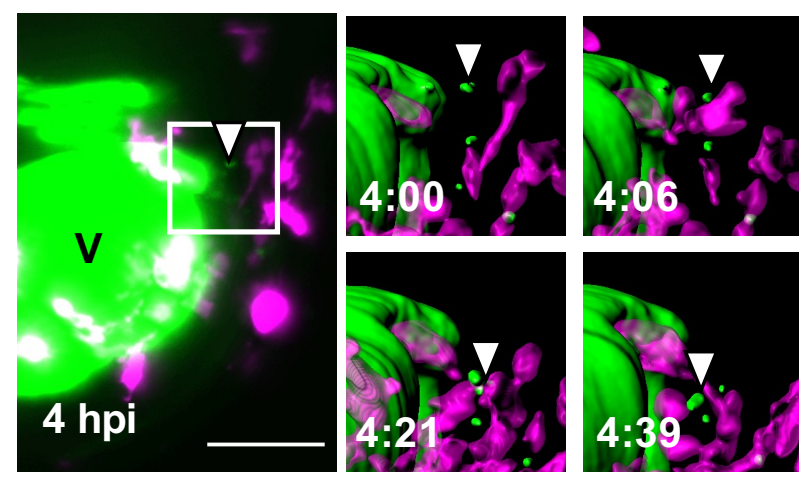

d Tg(myl7:mKateCAAX;myl7:h2b-GFP)

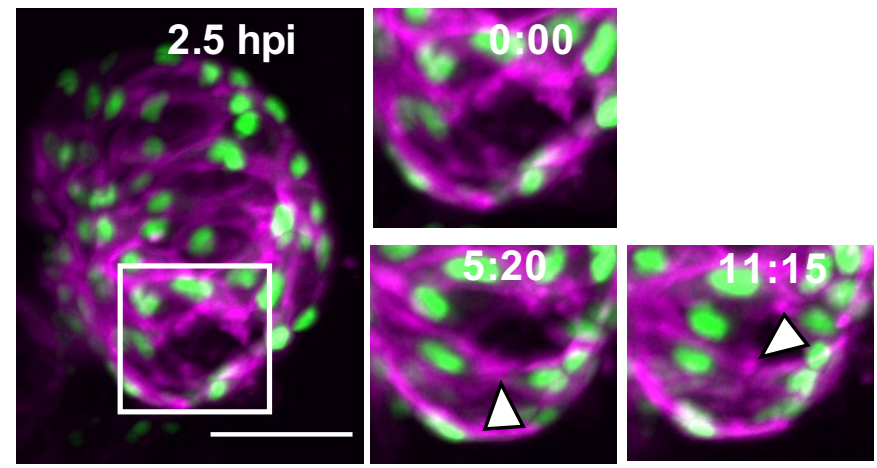


1054 Supplementary figure 2: Heartbeat-synchronised lightsheet-acquired time-lapse 1055 stills

1056 (a) Time-lapse stills of injured $\operatorname{Tg}($ myl7:h2b-GFP;myl7:mKateCAAX) ventricles 1057 imaged from 1 hpi. GFP intensity show by heat LUT, white arrowhead = apoptotic 1058 cardiomyocyte/condensing nucleus, white box $=$ zoom panel. (b) Time-lapse stills of 1059 injured Tg(myl7:h2b-GFP;myl7:mKateCAAX;mpeg1:GFP) ventricles imaged from 1 1060 hpi by heart-synchronised light-sheet imaging. Round GFPlow = cardiomyocyte nuclei 1061 and stellate GFPhigh =macrophages. Cyan arrowheads = Necrotic cardiomyocyte 1062 nuclei and white arrowheads = expelled necrotic cardiomyocyte, white box = zoom 1063 panel. (c) Time-lapse stills of an injured Tg(myl7:GFP;mpeg1:mCherry) ventricle from 10644 hpi where the full size panel has high gain in the GFP channel to highlight GFPlow 1065 myocardial debris and zoom panels (area indicated by white box) are surface rendered 1066 to highlight removal of myocardium (green) by macrophages (magenta). $V=$ high gain 1067 ventricle, white arrowhead = myocardial debris. (d) Time-lapse stills of an injured $1068 T g(m y l 7: m K a t e C A A X ; m y l 7: h 2 b-G F P)$ ventricle from $2.5 \mathrm{hpi}$. White box = zoom panel, 1069 white arrowheads $=$ cell-cell junctions 

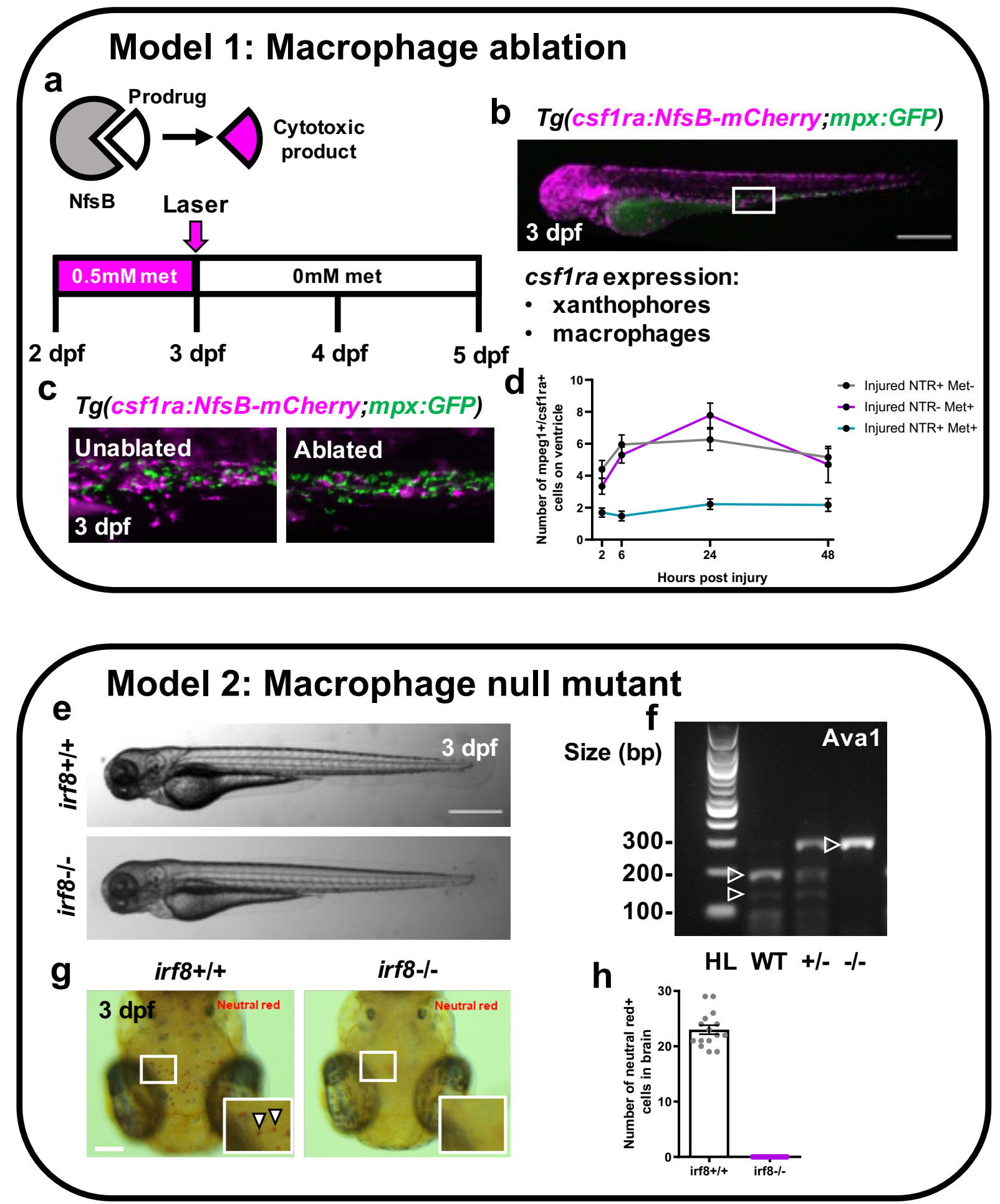

1073 Supplementary figure 3: Macrophages can be pharmacologically ablated or

1074 developmentally blocked genetically.

1075 (a) Schematic illustrating how nitroreductase enzyme 'NfsB' catabolises prodrug 1076 'metronidazole' to form a cytotoxic biproduct. (b) Representative epifluorescence 1077 image of a Tg(csf1ra:NfsB-mCherry;mpx:GFP ) 3 dpf larva (abbreviated to 1078 Tg(csf1ra:mCherry;mpx:GFP) in all panels), white box = caudal haematopoietic tissue, 
magenta $=$ macrophages and green $=$ neutrophils $(\mathrm{CHT})(\mathrm{c})$ Representative images of ablated and unablated macrophages in the $\mathrm{CHT}$, size and location indicated in (b)) in

1081 Tg(csf1ra:mCherry;mpx:GFP) 3dpf larvae. Macrophages are ablated and only 1082 apoptotic bodies remain but not neutrophils are unaffected. (d) Quantification of 1083 macrophages at standard timepoints, marked by either mpeg1 or csfr1a on the injured 1084 ventricle in each of the NTR=metronidazole ablation model's treatment groups 1085 NTR+Met-, NTR-Met+ and NTR+Met+. Macrophage ablation can be seen to abolish 1086 the macrophage response (e) Representative brightfield images of $\mathrm{irf}^{+/+}$and $\mathrm{irf8}^{-/-}$ larvae at $3 \mathrm{dpf}$. (f) Representative 1\% agarose gel displaying Ava1 restriction digest band pattern for WT, irf8 heterozygous and homozygous mutants. (g) Representative dorsal view brightfield image of 3 dpf larval heads stained with neutral red vital dye with white zoom panel highlighting stained macrophages (microglia) (red) in irf8+/+ but not irf8 $/$ larvae. (h) Quantification of the number of neutral red positive stained cells (macrophages/microglia) in larval brains of $i r f 8^{+/+}$and $i r f 8^{-/-}$at $3 \mathrm{dpf}$ showing $\mathrm{irfr}^{-/-}$ larvae to be macrophage-null. Scale bar $=500 \mu \mathrm{m}(\mathrm{b} \& \mathrm{e}), 100 \mu \mathrm{m}(\mathrm{g})$.
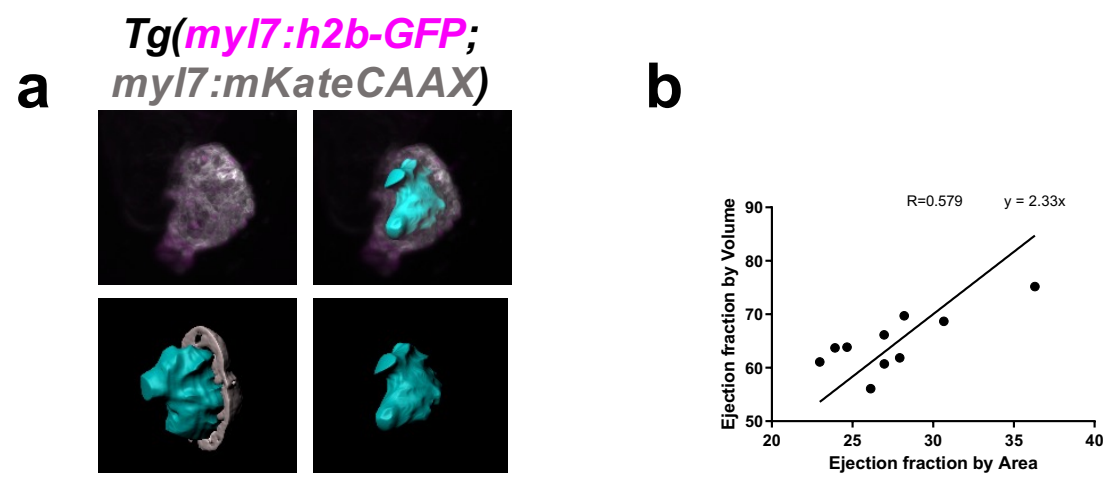

1098 Supplementary figure 4: Ejection fraction by area is proportional to ejection 1099 fraction by volume.

1100 (a) Representative IMARIS-generated image showing a rendered ventricular 1101 myocardium (grey render), rendered chamber volume (cyan) and MIP of 3D heartbeatsynchronised LSFM scan of a $3 \mathrm{dpf}$ heart (ventricle) in diastole. Image acquired from a Tg(myl7:h2b-GFP;myl7:mKateCAAX) larva. (b) Quantification of ejection fraction by area (calculated from diastolic and systolic lateral brightfield images) and by volume 
1105 (calculated from surface renders of luminal volumes in diastole and systole) for $n=10$ 1106 fish

a

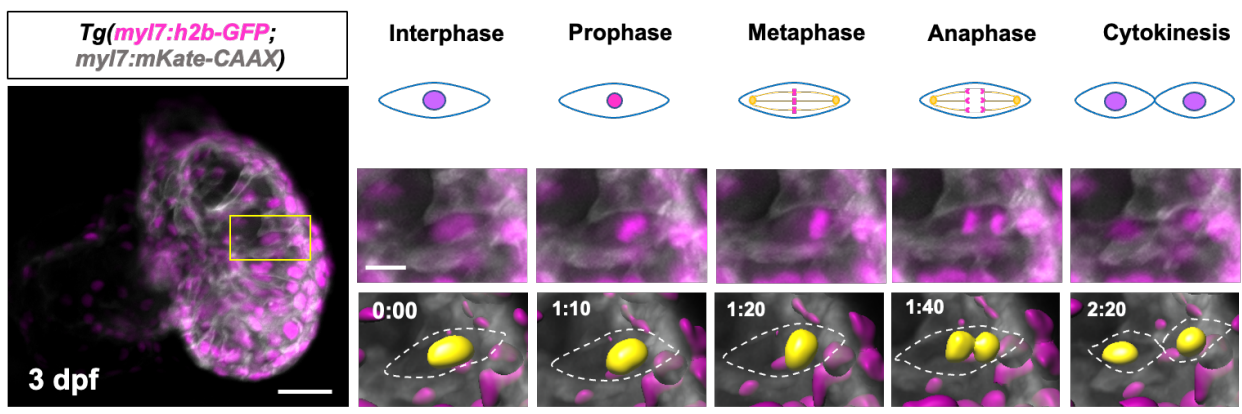

b
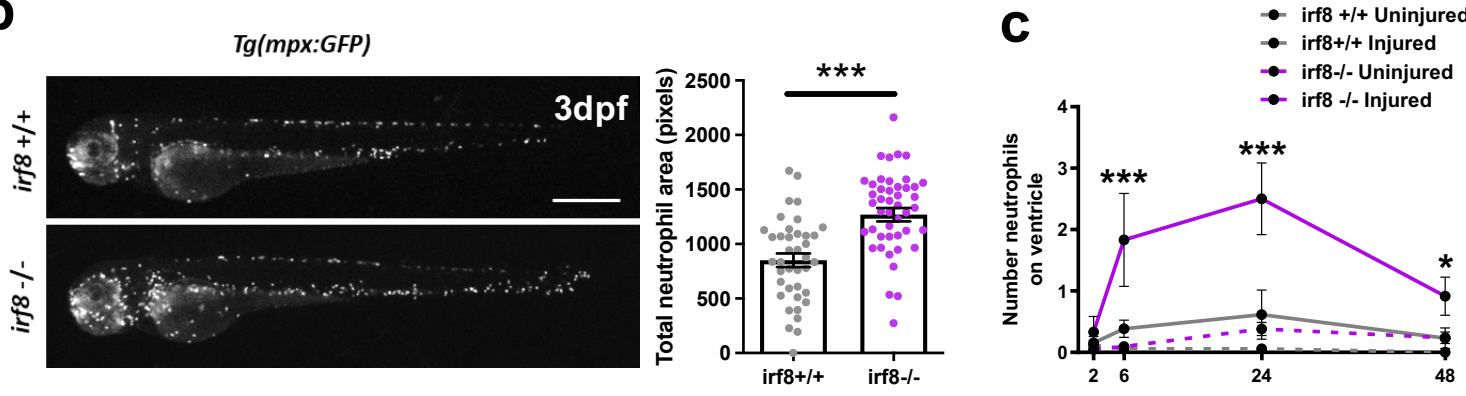

Hours post-injury

d

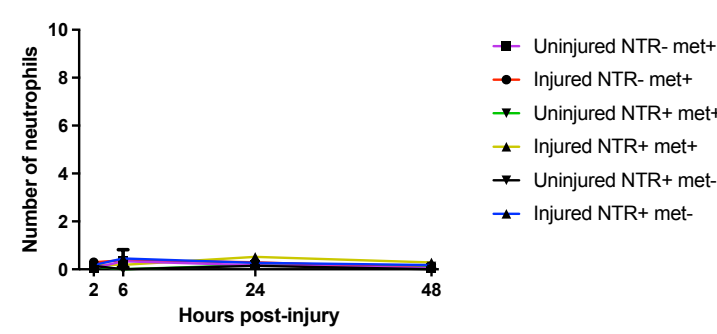

e

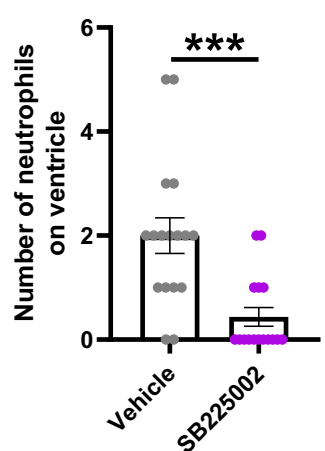

$\mathbf{f}$

Tg(myl7:mkateCAAX;

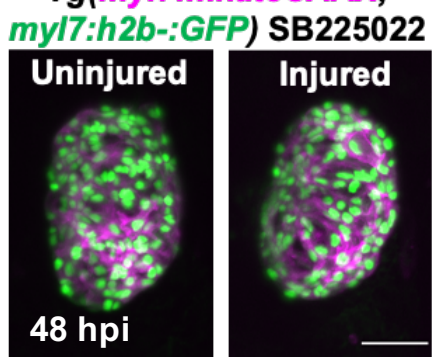

g

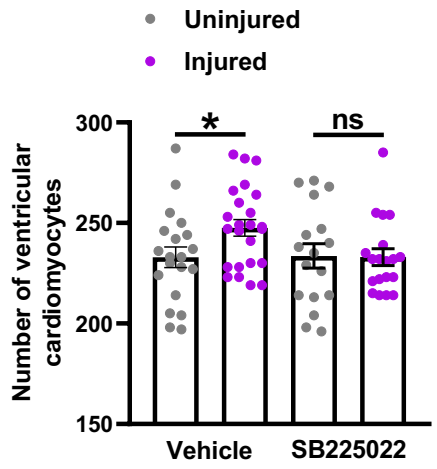

1109 Supplementary figure 5: irf8 ${ }^{-/-}$larvae have a larger neutrophil response to 1110 cardiac injury than irf8 ${ }^{+/+}$.

1111 (a) Representative timepoint images from heartbeat-synchronised LSFM time-lapse

1112 of a laser-injured $3 \mathrm{dpf} T g(m y l 7: h 2 b-G F P ; m y l 7: m K a t e C A A X)$ larva showing an 
1113 example of each phase of complete cell division of a single cardiomyocyte, typical of 1114 larval hearts. Yellow box $=$ zoom panel; left scale bar $=30 \mu \mathrm{m}$; right scale bar $=10$ $1115 \mu \mathrm{m}$. Timestamps post-injury indicated in figure. (b) Representative whole larva

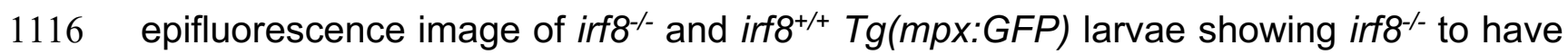
1117 greater global neutrophil numbers (scale bar $=500 \mu \mathrm{m}$ ), quantified in the graph (right). $1118{ }^{* * *} p \leq 0.001$. t test, $n=39-46$. (c) Quantification of neutrophil numbers at the ventricle in 1119 uninjured and injured irf $^{+/+}$and irf8 ${ }^{-/-}$larvae at the standard laser-injury model 1120 timepoints, showing irf8 ${ }^{-/}$larvae to have a significantly greater neutrophil response. $1121 \mathrm{n}=17-25$. (d) Quantification of neutrophil numbers at the ventricle in uninjured and 1122 injured NTR-met+, NTR+met+ and NTR+met- larvae at the standard laser-injury 1123 model timepoints. All metronidazole-nitroreductase treatment groups to have a 1124 minimal neutrophil response and therefore no neutrophil compensation in the 1125 macrophage ablated group NTR+met+, $n=17-24$. (e) Quantification of the number of 1126 recruited neutrophils at the injured ventricle in at $24 \mathrm{hpi}$ in $\operatorname{Tg}(m y / 7: \mathrm{h} 2 \mathrm{~b}$ 1127 GFP;myl7:mKateCAAX) larvae bathed in vehicle or SB225002 from -2 to +24 hpi 1128 showing SB225002 to significantly reduce neutrophil number, $n=17$. (f) Representative 1129 light-sheet acquired images of uninjured and injured irf8 ${ }^{-/} \operatorname{Tg}$ (myl7:h2b1130 GFP;myl7:mKateCAAX) ventricles at $48 \mathrm{hpi}$ following treatment with SB225002 from $1131-2$ to $+24 \mathrm{hpi}$, scale bar $=50 \mu \mathrm{m}$. (g) Quantification of ventricular cardiomyocyte 1132 number in uninjured and injured irf8 ${ }^{-/} \mathrm{Tg}($ myl7:h2b-GFP;myl7:mKateCAAX) ventricles 1133 at 48 hpi following treatment with vehicle or SB225002 -2 to $24 \mathrm{hpi}, \mathrm{n}=17-20,{ }^{*} p \leq 0.05$ $1134 \mathrm{t}$ test 
a

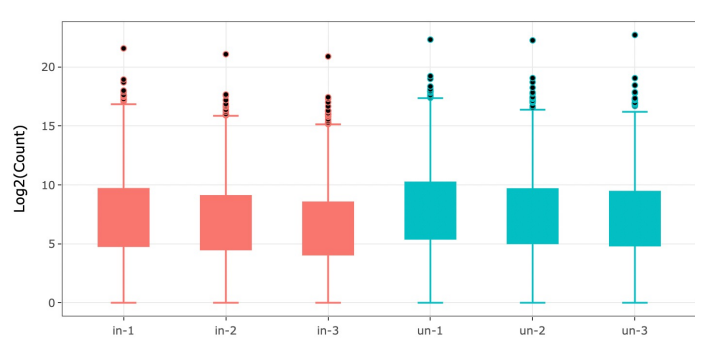

b

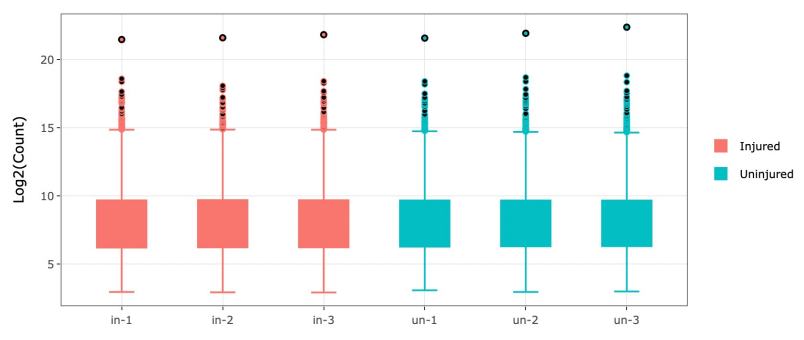

d
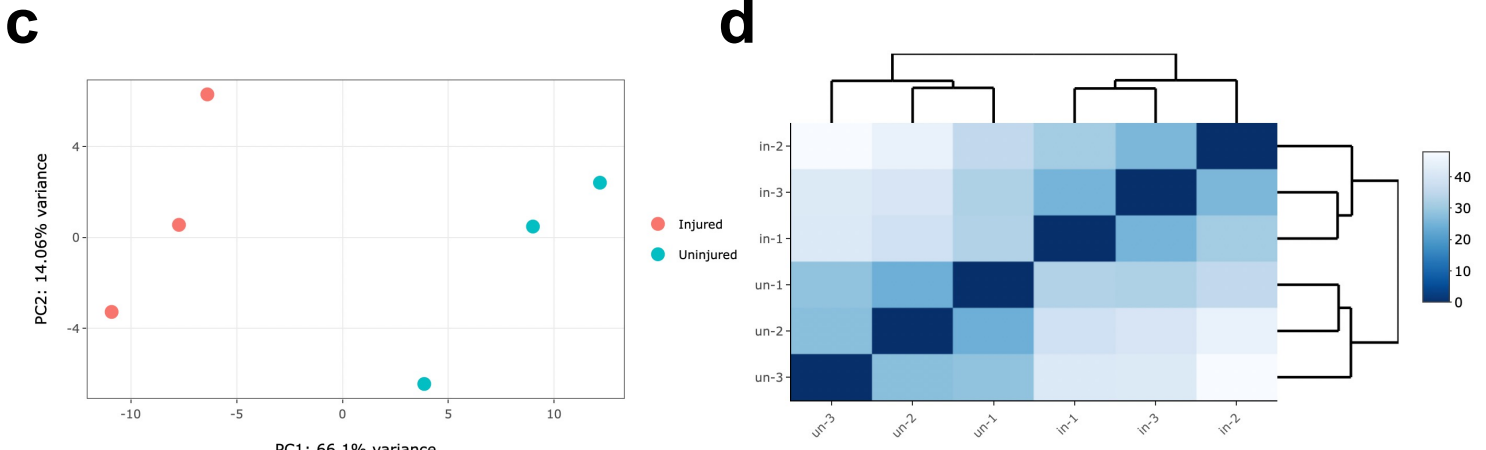

e

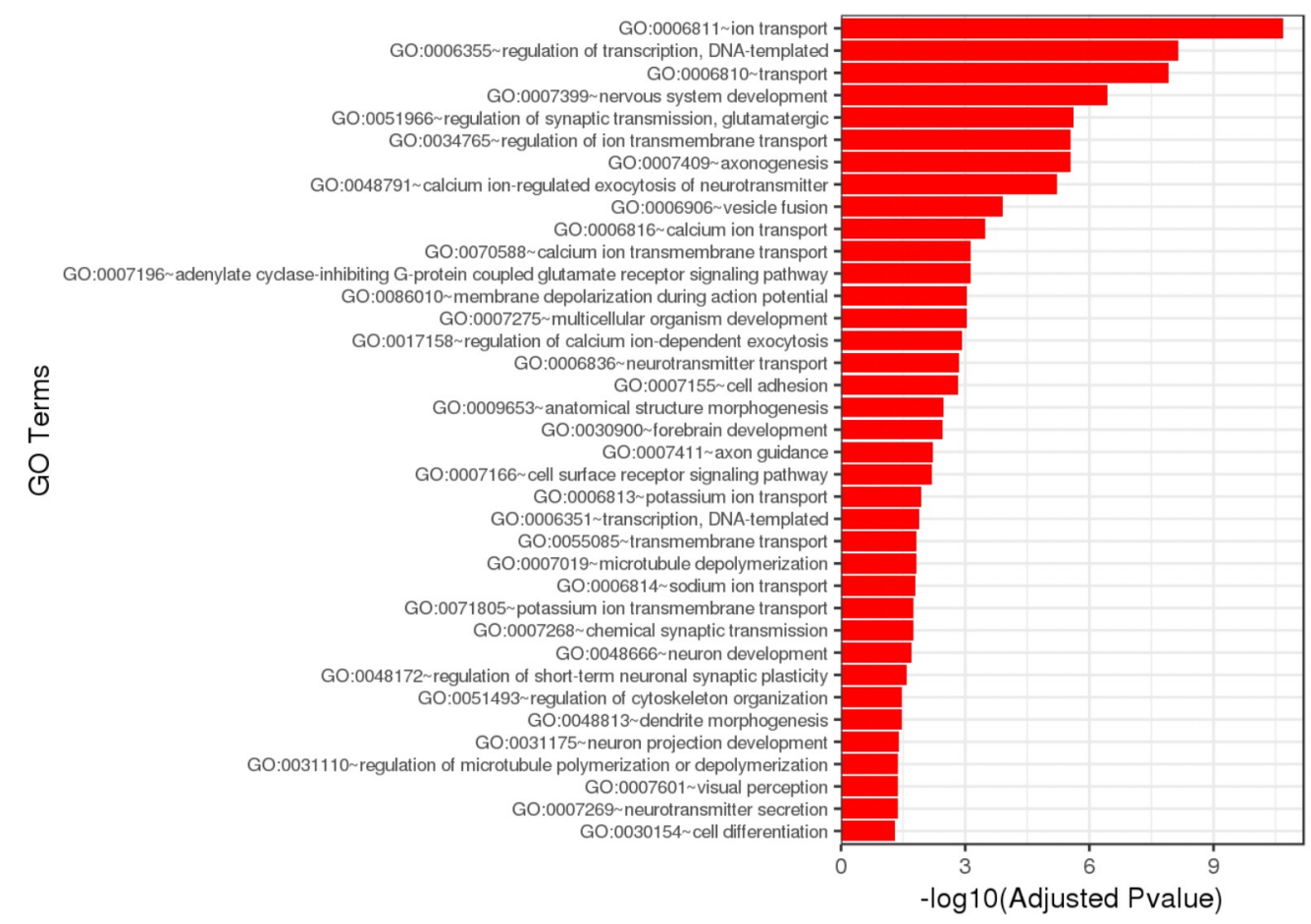

1137 Supplementary figure 6: Bulk RNAseq analysis of uninjured and injured larval

1138 hearts

1139 (a) Box plot illustrating the distribution of reads before (a) and after normalisation (b)

1140 Principal component analysis of samples, illustrating relative intragroup sample 
1141 similarity. (c) Distance matrix illustrating pairwise sample similarity. (e) Plot showing

1142 gene ontology terms that were significantly enriched by Fishers exact test for 1143 significantly (padj<0.05) differentially expressed genes between uninjured and injured 1144 hearts at $48 \mathrm{hpi}$.

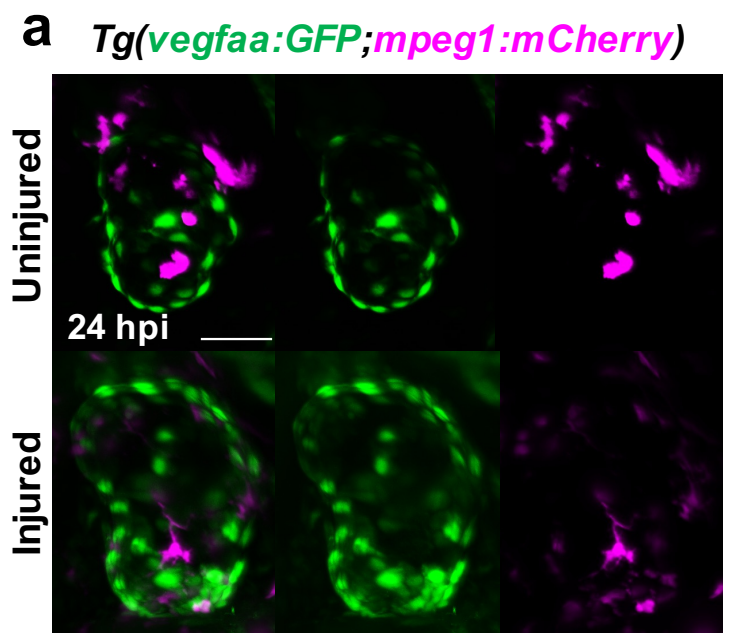

b Tg(vegfaa:GFP;mpx:mCherry)

C

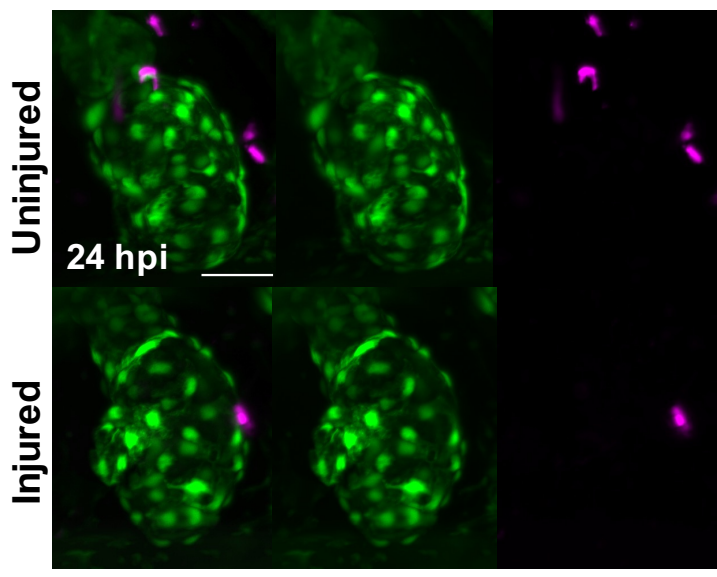

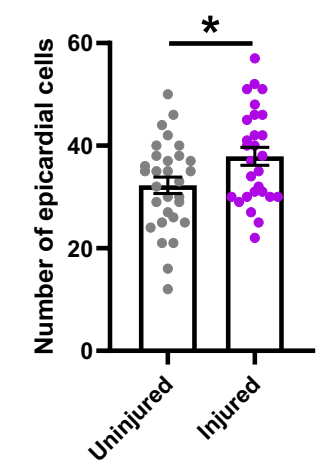

C d

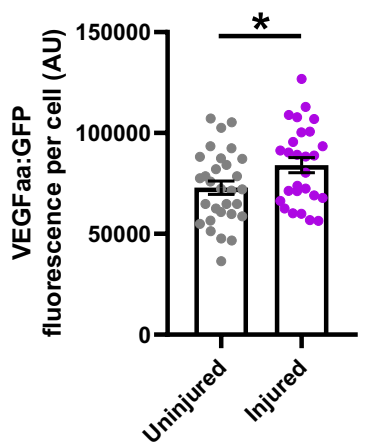

e

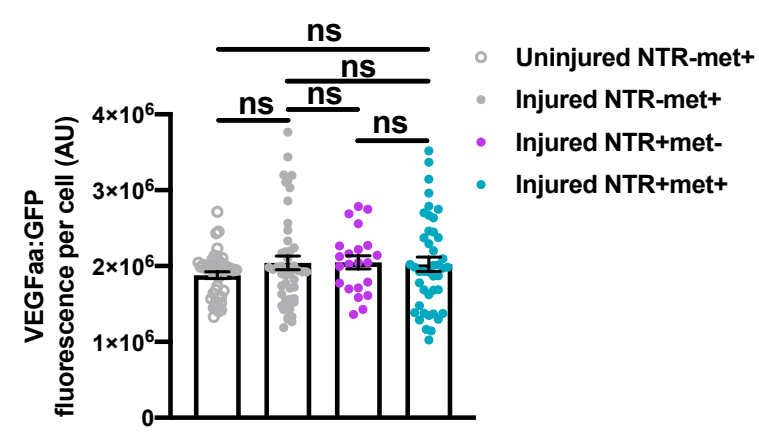

1147 Supplementary figure 7: vegfaa:GFP expression does not colocalize with 1148 macrophages or neutrophils following larval heart injury.

1149 (a) Representative LSFM image of an injured Tg(vegfaa:GFP;mpeg1:mCherry) heart 115024 hpi showing vegfaa:GFP expression only in the epicardium and not in 
macrophages, scale bar $=100 \mu \mathrm{m}$. (b) Representative LSFM image of an injured

1152 Tg(vegfaa:GFP;mpx:mCherry) heart 24 hpi showing VEGFaa expression only in the 1153 epicardium and not in neutrophils, scale bar $=100 \mu \mathrm{m}$. (c) Quantification of the number 1154 of epicardial cells, as marked by vegfaa:GFP, on injured ventricles at $48 \mathrm{hpi}$ in 1155 uninjured and injured larvae, $n=30$. ${ }^{*} p \leq 0.05 \mathrm{t}$ test (d) Quantification of the average 1156 vegfaa:GFP expression of epicardial cells per cell, on injured ventricles at 48 hpi in 1157 uninjured and injured larvae, $n=30 .{ }^{*} p \leq 0.05 \mathrm{t}$ test (e) Quantification of average vegfaa:GFP fluorescence per cell in metronidazole-nitroreductase ablation model groups at $48 \mathrm{hpi}, \mathrm{n}=22-44$. One-way ANOVA followed by Holm-Sidak's multiple comparisons Post-hoc test.

\section{References}

1. Ohnmacht, J., Yang, Y., Maurer, G. W., Barreiro-Iglesias, A., Tsarouchas, T.

3. Bensimon-Brito, A., Ramkumar, S., Boezio, G. L. M., Guenther, S., Kuenne, M., Wehner, D., Sieger, D., Becker, C. G. \& Becker, T. Spinal motor neurons are regenerated after mechanical lesion and genetic ablation in larval zebrafish. Dev. 143, 1464-1474 (2016).

2. Becker, C. G., Lieberoth, B. C., Morellini, F., Feldner, J., Becker, T. \& Schachner, M. L1.1 is involved in spinal cord regeneration in adult zebrafish. $J$. Neurosci. 24, 7837-7842 (2004).

C., Helker, C. S. M., Sánchez-Iranzo, H., lloska, D., Piesker, J., Pullamsetti, S., Mercader, N., Beis, D. \& Stainier, D. Y. R. TGF- $\beta$ Signaling Promotes Tissue Formation during Cardiac Valve Regeneration in Adult Zebrafish. Dev. Cell $\mathbf{0}$, (2019).

4. Sander, V., Davidson, A. J., Sander, V. \& Davidson, A. J. Kidney Injury and Regeneration in Zebrafish. Semin. Nephrol. 34, 437-444 (2014).

5. Basu Mallik, S., Jayashree, B. S. \& Shenoy, R. R. Epigenetic modulation of macrophage polarization- perspectives in diabetic wounds. J. Diabetes Complications 0, (2018).

6. González-Rosa, J. M., Martín, V., Peralta, M., Torres, M. \& Mercader, N. Extensive scar formation and regression during heart regeneration after cryoinjury in zebrafish. Development 138, 1663-1674 (2011). 
1185 7. Leuschner, F., Rauch, P. J., Ueno, T., Gorbatov, R., Marinelli, B., Lee, W. W., Dutta, P., Wei, Y., Robbins, C., Iwamoto, Y., Sena, B., Chudnovskiy, A., Panizzi, P., Keliher, E., Higgins, J. M., Libby, P., Moskowitz, M. A., Pittet, M. J., Swirski, F. K., Weissleder, R. \& Nahrendorf, M. Rapid monocyte kinetics in acute myocardial infarction are sustained by extramedullary monocytopoiesis. J. Exp. Med. 209, 123-37 (2012).

8. Murry, C. E., Reinecke, H. \& Pabon, L. M. Regeneration Gaps. Observations on Stem Cells and Cardiac Repair. Journal of the American College of Cardiology vol. 47 1777-1785 (2006).

9. Bergmann, O., Zdunek, S., Felker, A., Salehpour, M., Alkass, K., Bernard, S., Sjostrom, S. L., Szewczykowska, M., Jackowska, T., dos Remedios, C., Malm, T., Andrä, M., Jashari, R., Nyengaard, J. R., Possnert, G., Jovinge, S., Druid, H. \& Frisén, J. Dynamics of Cell Generation and Turnover in the Human Heart. Cell 161, 1566-1575 (2015).

10. Pfeffer, M. A. \& Braunwald, E. Ventricular remodeling after myocardial infarction. Experimental observations and clinical implications. Circulation 81, 1161-72 (1990).

11. Richardson, W. J., Clarke, S. A., Quinn, T. A. \& Holmes, J. W. Physiological Implications of Myocardial Scar Structure. Compr. Physiol. 5, 1877-909 (2015).

12. Jopling, C., Sleep, E., Raya, M., Martí, M., Raya, A. \& Belmonte, J. C. I. Zebrafish heart regeneration occurs by cardiomyocyte dedifferentiation and proliferation. Nature 464, 606-609 (2010).

13. Kikuchi, K., Holdway, J. E., Werdich, A. A., Anderson, R. M., Fang, Y., Egnaczyk, G. F., Evans, T., MacRae, C. A., Stainier, D. Y. R. \& Poss, K. D. Primary contribution to zebrafish heart regeneration by gata4+ cardiomyocytes. Nature 464, 601-605 (2010).

14. González-rosa, J. M., Martín, V., Peralta, M., Torres, M. \& Mercader, N. Extensive scar formation and regression during heart regeneration after cryoinjury in zebrafish. 1674, 1663-1674 (2011).

15. Aurora, A. B., Porrello, E. R., Tan, W., Mahmoud, A. I., Hill, J. A., Bassel-duby, R., Sadek, H. A. \& Olson, E. N. Macrophages are required for neonatal heart regeneration. 124, (2014).

16. Godwin, J. W., Debuque, R., Salimova, E. \& Rosenthal, N. A. Heart 
regeneration in the salamander relies on macrophage-mediated control of fibroblast activation and the extracellular landscape. npj Regen. Med. 2, 22 (2017).

1222

17. Lai, S.-L., Marín-Juez, R., Moura, P. L., Kuenne, C., Lai, J. K. H., Tsedeke, A. T., Guenther, S., Looso, M. \& Stainier, D. Y. Reciprocal analyses in zebrafish and medaka reveal that harnessing the immune response promotes cardiac regeneration. Elife 6, (2017).

18. Tsarouchas, T. M., Wehner, D., Cavone, L., Munir, T., Keatinge, M., Lambertus, M., Underhill, A., Barrett, T., Kassapis, E., Ogryzko, N., Feng, Y., van Ham, T. J., Becker, T. \& Becker, C. G. Dynamic control of proinflammatory cytokines II- $1 \beta$ and Tnf- $\alpha$ by macrophages in zebrafish spinal cord regeneration. Nat. Commun. 9, 4670 (2018).

19. Nahrendorf, M., Swirski, F. K., Aikawa, E., Stangenberg, L., Wurdinger, T., Figueiredo, J.-L., Libby, P., Weissleder, R. \& Pittet, M. J. The healing myocardium sequentially mobilizes two monocyte subsets with divergent and complementary functions. J. Exp. Med. 204, 3037-3047 (2007).

20. Sager, H. B., Hulsmans, M., Lavine, K. J., Moreira, M. B., Heidt, T., Courties, G., Sun, Y., Iwamoto, Y., Tricot, B., Khan, O. F., Dahlman, J. E., Borodovsky, A., Fitzgerald, K., Anderson, D. G., Weissleder, R., Libby, P., Swirski, F. K. \& Nahrendorf, M. Proliferation and Recruitment Contribute to Myocardial Macrophage Expansion in Chronic Heart Failure. Circ. Res. 119, 853-64 (2016).

21. Nahrendorf, M. \& Swirski, F. K. Monocyte and Macrophage Heterogeneity in the Heart. 02114, 1624-1634 (2013).

22. Lavin, Y., Winter, D., Blecher-Gonen, R., David, E., Keren-Shaul, H., Merad, M., Jung, S. \& Amit, I. Tissue-Resident Macrophage Enhancer Landscapes Are Shaped by the Local Microenvironment. Cell 159, 1312-1326 (2014).

23. Sanz-Morejón, A., García-Redondo, A. B., Reuter, H., Marques, I. J., Bates, T., Galardi-Castilla, M., Große, A., Manig, S., Langa, X., Ernst, A., Piragyte, I., Botos, M.-A., González-Rosa, J. M., Ruiz-Ortega, M., Briones, A. M., Salaices, M., Englert, C. \& Mercader, N. Wilms Tumor 1b Expression Defines a Proregenerative Macrophage Subtype and Is Required for Organ Regeneration in the Zebrafish. Cell Rep. 28, 1296-1306.e6 (2019).

24. Nguyen-Chi, M., , B'eryl Laplace-BuilheTravnickova, J., Luz-crawford, P., 
Tejedor, G., Phan, Q. T., Duroux-richard, I., Levraud, J., Kissa, K., Lutfalla, G., Jorgensen, C., Djouad, F., Laplace-Builhe, B., Travnickova, J., Luz-crawford, P., Tejedor, G., Phan, Q. T., Duroux-richard, I., Levraud, J., Kissa, K., Lutfalla, G., Jorgensen, C. \& Djouad, F. Identification of polarized macrophage subsets in zebrafish. Elife 4, 1-14 (2015).

25. Matrone, G., Taylor, J., Wilson, K., ... J. B.-I. journal of \& 2013, undefined. Laser-targeted ablation of the zebrafish embryonic ventricle: a novel model of cardiac injury and repair. Elsevier.

26. Kaveh, A., Bruton, F. A., Buckley, C., Oremek, M. E. M., Tucker, C. S., Mullins, J. J., Taylor, J. M., Rossi, A. G. \& Denvir, M. A. Live Imaging of Heart Injury in Larval Zebrafish Reveals a Multi-Stage Model of Neutrophil and Macrophage Migration. Front. Cell Dev. Biol. 8, 579943 (2020).

27. Shiau, C. E., Kaufman, Z., Meireles, A. M. \& Talbot, W. S. Differential Requirement for irf8 in Formation of Embryonic and Adult Macrophages in Zebrafish. PLoS One 10, 1-15 (2015).

30. Taylor, J. M., Nelson, C. J., Bruton, F. A., Baghbadrani, A. K., Buckley, C.,

32. Pisharath, H., Rhee, J. M., Swanson, M. A., Leach, S. D. \& Parsons, M. J.

28. Hume, D. A., Wollscheid-Lengeling, E., Rojo, R. \& Pridans, C. The evolution of the macrophage-specific enhancer (Fms intronic regulatory element) within the CSF1R locus of vertebrates. Sci. Rep. 7, 1-10 (2017).

29. Bevan, L., Lim, Z. W., Venkatesh, B., Riley, P. R., Martin, P. \& Richardson, R. J. Specific macrophage populations promote both cardiac scar deposition and subsequent resolution in adult zebrafish. Cardiovasc. Res. (2019) doi:10.1093/cvr/cvz221.

Tucker, C. S., Rossi, A. G., Mullins, J. J. \& Denvir, M. A. Adaptive prospective optical gating enables day-long 3D time-lapse imaging of the beating embryonic zebrafish heart. Nat. Commun. 10,1-15 (2019).

31. Krijnen, P. A. J., Nijmeijer, R., Meijer, C. J. L. M., Visser, C. A., Hack, C. E. \& Niessen, H. W. M. Apoptosis in myocardial ischaemia and infarction. J. Clin. Pathol. 55, 801-11 (2002). Targeted ablation of beta cells in the embryonic zebrafish pancreas using $\mathrm{E}$. coli nitroreductase. Mech. Dev. 124, 218-229 (2007).

33. Karra, R., Foglia, M. J., Choi, W.-Y., Belliveau, C., DeBenedittis, P. \& Poss, K. D. Vegfaa instructs cardiac muscle hyperplasia in adult zebrafish. Proc. Natl. 
Acad. Sci. U. S. A. 201722594 (2018) doi:10.1073/pnas.1722594115.

34. Chimote, G., Sreenivasan, J., Pawar, N., Subramanian, J., Sivaramakrishnan, H. \& Sharma, S. Comparison of effects of anti-angiogenic agents in the

36. Honkoop, H., Bakker, D. E. de, Aharonov, A., Kruse, F., Shakked, A., Nguyen, zebrafish efficacy-toxicity model for translational anti-angiogenic drug discovery. Drug Des. Devel. Ther. 8, 1107-1123 (2014).

35. Gemberling, M., Karra, R., Dickson, A. L. \& Poss, K. D. Nrg1 is an injuryinduced cardiomyocyte mitogen for the endogenous heart regeneration program in zebrafish. 1-17 (2015) doi:10.7554/eLife.05871.

P. D., Heus, C. de, Garric, L., Muraro, M. J., Shoffner, A., Tessadori, F., Peterson, J. C., Noort, W., Bertozzi, A., Weidinger, G., Posthuma, G., Grün, D., Laarse, W. J. van der, Klumperman, J., Jaspers, R. T., Poss, K. D., Oudenaarden, A. van, Tzahor, E., Bakkers, J., de Bakker, D. E., Aharonov, A., Kruse, F., Shakked, A., Nguyen, P. D., de Heus, C., Garric, L., Muraro, M. J., Shoffner, A., Tessadori, F., Peterson, J. C., Noort, W., Bertozzi, A., Weidinger, G., Posthuma, G., Grun, D., van der Laarse, W. J., Klumperman, J., Jaspers, R. T., Poss, K. D., van Oudenaarden, A., Tzahor, E. \& Bakkers, J. Single-cell analysis uncovers that metabolic reprogramming by ErbB2 signaling is essential for cardiomyocyte proliferation in the regenerating heart. Elife 8, (2019).

37. Uribe, V., Ramadass, R., Dogra, D., Rasouli, S. J., Gunawan, F., Nakajima, H., Chiba, A., Reischauer, S., Mochizuki, N. \& Stainier, D. Y. R. In vivo analysis of cardiomyocyte proliferation during trabeculation. (2018) doi:10.1242/dev.164194.

38. Zhao, L., Ben-Yair, R., Burns, C. E. \& Burns, C. G. Endocardial Notch Signaling Promotes Cardiomyocyte Proliferation in the Regenerating Zebrafish Heart through Wnt Pathway Antagonism. Cell Rep. 26, 546-554.e5 (2019).

39. Dovey, H. F., John, V., Anderson, J. P., Chen, L. Z., De Saint Andrieu, P., Fang, L. Y., Freedman, S. B., Folmer, B., Goldbach, E., Holsztynska, E. J., Hu, K. L., Johnson-Wood, K. L., Kennedy, S. L., Kholodenko, D., Knops, J. E., Latimer, L. H., Lee, M., Liao, Z., Lieberburg, I. M., Motter, R. N., Mutter, L. C., Nietz, J., Quinn, K. P., Sacchi, K. L., Seubert, P. A., Shopp, G. M., Thorsett, E. D., Tung, J. S., Wu, J., Yang, S., Yin, C. T., Schenk, D. B., May, P. C., Altstiel, L. D., Bender, M. H., Boggs, L. N., Britton, T. C., Clemens, J. C., Czilli, D. L., 
Dieckman-McGinty, D. K., Droste, J. J., Fuson, K. S., Gitter, B. D., Hyslop, P. A., Johnstone, E. M., Li, W. Y., Little, S. P., Mabry, T. E., Miller, F. D., Ni, B., Nissen, J. S., Porter, W. J., Potts, B. D., Reel, J. K., Stephenson, D., Su, Y., Shipley, L. A., Whitesitt, C. A., Yin, T. \& Audia, J. E. Functional gammasecretase inhibitors reduce beta-amyloid peptide levels in brain. $J$. Neurochem. 76, 173-181 (2001).

40. Micchelli, C. A., Esler, W. P., Kimberly, W. T., Jack, C., Berezovska, O., Kornilova, A., Hyman, B. T., Perrimon, N. \& Wolfe, M. S. Gammasecretase/presenilin inhibitors for Alzheimer's disease phenocopy Notch mutations in Drosophila. FASEB J. 17, 79-81 (2003).

41. Geling, A., Steiner, H., Willem, M., Bally-Cuif, L. \& Haass, C. A y-secretase inhibitor blocks Notch signaling in vivo and causes a severe neurogenic phenotype in zebrafish. EMBO Rep. 3, 688-694 (2002).

42. Lyons, D. A., Pogoda, H. M., Voas, M. G., Woods, I. G., Diamond, B., Nix, R., Arana, N., Jacobs, J. \& Talbot, W. S. erbb3 and erbb2 are essential for Schwann cell migration and myelination in zebrafish. Curr. Biol. 15, 513-524 (2005).

43. Samsa, L. A., Givens, C., Tzima, E., Stainier, D. Y. R., Qian, L. \& Liu, J. Cardiac contraction activates endocardial Notch signaling to modulate chamber maturation in zebrafish. Development 142, 4080-4091 (2015).

44. Gálvez-Santisteban, M., Chen, D., Zhang, R., Serrano, R., Nguyen, C., Zhao, L., Nerb, L., Masutani, E. M., Vermot, J., Burns, C. G., Burns, C. E., del Álamo, J. C. \& Chi, N. C. Hemodynamic-mediated endocardial signaling controls in vivo myocardial reprogramming. Elife 8, (2019).

45. Kuil, L. E., Oosterhof, N., Ferrero, G., Mikulášová, T., Hason, M., Dekker, J., Rovira, M., van der Linde, H. C., van Strien, P. M. H., de Pater, E., Schaaf, G., Bindels, E. M. J., Wittamer, V. \& van Ham, T. J. Zebrafish macrophage developmental arrest underlies depletion of microglia and reveals Csf1rindependent metaphocytes. Elife 9, (2020).

47. Koth, J., Wang, X., Killen, A. C., Stockdale, W. T., Potts, H. G., Jefferson, A.,

46. Lin, X., Zhou, Q., Zhao, C., Lin, G., Xu, J. \& Wen, Z. An Ectoderm-Derived Myeloid-like Cell Population Functions as Antigen Transporters for Langerhans Cells in Zebrafish Epidermis. Dev. Cell 49, 605-617.e5 (2019). Bonkhofer, F., Riley, P. R., Patient, R. K., Göttgens, B. \& Mommersteeg, M. T. 
M. Runx1 promotes scar deposition and inhibits myocardial proliferation and survival during zebrafish heart regeneration. Development 147, (2020).

48. Herrgen, L., Voss, O. P. \& Akerman, C. J. Calcium-Dependent Neuroepithelial Contractions Expel Damaged Cells from the Developing Brain. Dev. Cell 31, 599-613 (2014).

1360

49. Leor, J., Palevski, D., Amit, U. \& Konfino, T. Seminars in Cell \& Developmental Biology Macrophages and regeneration: Lessons from the heart. Semin. Cell Dev. Biol. 58, 26-33 (2016).

50. Beisaw, A., Kuenne, C., Günther, S., Dallmann, J., Wu, C.-C., Bentsen, M., Looso, M. \& Stainier, D. AP-1 Contributes to Chromatin Accessibility to Promote Sarcomere Disassembly and Cardiomyocyte Protrusion during Zebrafish Heart Regeneration. Circ. Res. CIRCRESAHA.119.316167 (2020) doi:10.1161/CIRCRESAHA.119.316167.

51. Morikawa, Y., Zhang, M., Heallen, T., Leach, J., Tao, G., Xiao, Y., Bai, Y., Li, W., Willerson, J. T. \& Martin, J. F. Actin cytoskeletal remodeling with protrusion formation is essential for heart regeneration in Hippo-deficient mice. Sci. Signal. 8, (2015).

52. Marín-juez, R., Marass, M., Gauvrit, S., Rossi, A., Lai, S. \& Materna, S. C. Fast revascularization of the injured area is essential to support zebrafish heart regeneration. 113, (2016).

53. Stevens, S. M., von Gise, A., VanDusen, N., Zhou, B. \& Pu, W. T. Epicardium is required for cardiac seeding by yolk sac macrophages, precursors of resident macrophages of the adult heart. Dev. Biol. 413, 153-159 (2016).

54. Deniset, J. F., Belke, D., Lee, W.-Y., Weber, G. F., Fedak, P. W. M. \& Kubes, P. Gata6+ Pericardial Cavity Macrophages Relocate to the Injured Heart and Prevent Cardiac Fibrosis. Immunity 51, 131-140.e5 (2019).

55. Zhao, L., Ben-Yair, R., Burns, C. E. G. \& Burns, C. E. G. Endocardial Notch Signaling Promotes Cardiomyocyte Proliferation in the Regenerating Zebrafish Heart through Wnt Pathway Antagonism. Cell Rep. 26, 546-554.e5 (2019).

56. Zhao, L., Borikova, A. L., Ben-Yair, R., Guner-Ataman, B., MacRae, C. A., Lee, R. T., Geoffrey Burns, C. \& Burns, C. E. Notch signaling regulates cardiomyocyte proliferation during zebrafish heart regeneration. Proc. Natl. Acad. Sci. U. S. A. 111, 1403-1408 (2014).

57. Raya, A., Koth, C. M., Büscher, D., Kawakami, Y., Itoh, T., Raya, R. M., 
Sternik, G., Tsai, H.-J., Rodríguez-Esteban, C. \& Izpisúa-Belmonte, J. C. Activation of Notch signaling pathway precedes heart regeneration in zebrafish. Proc. Natl. Acad. Sci. U. S. A. 100 Suppl 1, 11889-95 (2003).

58. Zhao, L., Ben-Yair, R., Burns, C. G. E. G. \& Burns, C. G. E. G. Endocardial Notch Signaling Promotes Cardiomyocyte Proliferation in the Regenerating Zebrafish Heart through Wnt Pathway Antagonism. Cell Rep. 26, 546-554.e5 (2019).

59. Gemberling, M., Karra, R., Dickson, A. L. \& Poss, K. D. Nrg1 is an injuryinduced cardiomyocyte mitogen for the endogenous heart regeneration program in zebrafish. Elife 4, (2015).

60. Huang, C. J., Tu, C. T., Hsiao, C. Der, Hsieh, F. J. \& Tsai, H. J. Germ-line transmission of a myocardium-specific GFP transgene reveals critical regulatory elements in the cardiac myosin light chain 2 promoter of zebrafish. Dev. Dyn. 228, 30-40 (2003).

63. Renshaw, S. A., Loynes, C. A., Trushell, D. M. I., Elworthy, S., Ingham, P. W.

61. Yoo, S. K., Deng, Q., Cavnar, P. J., Wu, Y. I., Hahn, K. M. \& Huttenlocher, A. Differential Regulation of Protrusion and Polarity by $\mathrm{PI}(3) \mathrm{K}$ during Neutrophil Motility in Live Zebrafish. Dev. Cell 18, 226-236 (2010).

62. Ellett, F., Pase, L., Hayman, J. W., Andrianopoulos, A. \& Lieschke, G. J. mpeg1 promoter transgenes direct macrophage-lineage expression in zebrafish. Blood 117, e49 (2011).

66. Gray, C., Loynes, C. A., Whyte, M. K. B., Crossman, D. C., Renshaw, S. A. \& \& Whyte, M. K. B. A transgenic zebrafish model of neutrophilic inflammation. Blood 108, 3976-3978 (2006).

64. Mickoleit, M., Schmid, B., Weber, M., Fahrbach, F. O., Hombach, S., Reischauer, S. \& Huisken, J. High-resolution reconstruction of the beating zebrafish heart. Nat. Methods 11, 919-922 (2014).

65. Lin, Y.-F., Swinburne, I. \& Yelon, D. Multiple influences of blood flow on cardiomyocyte hypertrophy in the embryonic zebrafish heart. Dev. Biol. 362, 242-253 (2012).

Chico, T. J. A. Simultaneous intravital imaging of macrophage and neutrophil behaviour during inflammation using a novel transgenic zebrafish. 811-819 (2011) doi:10.1160/TH10-08-0525.

67. Davison, J. M., Akitake, C. M., Goll, M. G., Rhee, J. M., Gosse, N., Baier, H., 

transgenic insertions for tissue-specific cell labeling and ablation in zebrafish. Dev. Biol. 304, 811-824 (2007).

68. Rottbauer, W., Saurin, A. J., Lickert, H., Shen, X., Burns, C. G., Wo, Z. G., Kemler, R., Kingston, R., Wu, C. \& Fishman, M. Reptin and pontin

69. Ninov, N., Borius, M. \& Stainier, D. Y. R. Different levels of Notch signaling regulate quiescence, renewal and differentiation in pancreatic endocrine progenitors. Development 139, 1557-1567 (2012).

70. Chi, N. C., Shaw, R. M., De Val, S., Kang, G., Jan, L. Y., Black, B. L. \& Stainier, D. Y. R. Foxn4 directly regulates tbx2b expression and atrioventricular canal formation. Genes Dev. 22, 734-739 (2008).

71. Choi, J., Dong, L., Ahn, J., Dao, D., Hammerschmidt, M. \& Chen, J. N. FoxH1 negatively modulates flk1 gene expression and vascular formation in zebrafish. Dev. Biol. 304, 735-744 (2007).

72. Kikuchi, K., Gupta, V., Wang, J., Holdway, J. E., Wills, A. A., Fang, Y. \& Poss, K. D. tcf21+ epicardial cells adopt non-myocardial fates during zebrafish heart development and regeneration. Development 138, 2895-902 (2011).

73. Mickoleit, M., Schmid, B., Weber, M., Fahrbach, F. O., Hombach, S., Reischauer, S. \& Huisken, J. High-resolution reconstruction of the beating zebrafish heart. Nat. Methods 11, 919-922 (2014).

74. Schindelin, J., Arganda-Carreras, I., Frise, E., Kaynig, V., Longair, M., Pietzsch, T., Preibisch, S., Rueden, C., Saalfeld, S., Schmid, B., Tinevez, J. Y., White, D. J., Hartenstein, V., Eliceiri, K., Tomancak, P. \& Cardona, A. Fiji: An open-source platform for biological-image analysis. Nature Methods vol. 9 676-682 (2012).

75. Burns, C. G. \& MacRae, C. A. Purification of hearts from zebrafish embryos. BioTechniques vol. 40 274-282 (2006).

Videos:

Videos 1-8 can be found deposited on Dropbox via the following link: 
https://www.dropbox.com/sh/xy5re3qvf4oc327/AAAVYj6SICxZBJGKKYQLNI6la?dl=

$1458 \underline{0}$

1459

1460 Video 1: LSFM-acquired heartbeat-synchronised time-lapse of a

1461 Tg(csf1ra:mCherry;mpeg1:GFP) heart showing macrophage heterogeneity following 1462 cardiac injury.

1463 Video 2: LSFM-acquired heartbeat-synchronised time-lapse of a

1464 Tg(mpeg1:mCherry;tnfa:GFP) heart showing macrophage plasticity following cardiac 1465 injury.

1466 Video 3: LSFM-acquired heartbeat-synchronised time-lapse of a $\operatorname{Tg}($ myl7:h2b-

1467 GFP;myl7:mKateCAAX) heart following cardiac injury showing cardiomyocyte

1468 apoptosis following injury.

1469 Video 4: LSFM-acquired heartbeat-synchronised time-lapse of a $\operatorname{Tg}($ myl7:h2b-

1470 GFP;mpeg1:GFP) heart injected with propdium iodide showing $\mathrm{PI}+$ cardiomyocyte

1471 expulsion following cardiac injury.

1472 Video 5: LSFM-acquired heartbeat-synchronised time-lapse of a

1473 Tg(myl7:GFP;mpeg1:mCherry) heart, 3D surface rendered, showing removal and

1474 internalization of myocardial debris by macrophages following injury.

1475 Video 6: LSFM-acquired heartbeat-synchronised time-lapse of a $\operatorname{Tg}($ myl7:GFP)

1476 heart, 3D surface rendered, showing budding and bridging of wound margin

1477 myocardium following injury.

1478 Video 7: LSFM-acquired heartbeat-synchronised time-lapse of a Tg(myl7:h2b-

1479 GFP;myl7:mKateCAAX) heart, showing budding and bridging of individual wound-

1480 margin cardiomyocytes following injury.

1481 Video 8: LSFM-acquired heartbeat-synchronised time-lapse of a $\operatorname{Tg}($ myl7:h2b-

1482 GFP;myl7:mKateCAAX) heart, showing cardiomyocyte cell division with nuclear

1483 division and cytokinesis. 